\title{
STRATIGRAPHIC SECTIONS OF THE PHOSPHORIA FORMATION IN
}

\section{IDAHO, 1949, PART 2}

This report concerns work done on behalf of the U. S. Atomic Energy Commission and is published with the permission of the Commission. 
UNITED STATES LEPARTMENT OF THE INTERIOR

Doug as McKay, Secretary

GEOLOGICAL SURVEY

W. E Wra her, Director

GEOLOGICAL SURVEY CIRCULAR 305

\section{STRATIGRAPHIC SECTIONS OF THE PHOSPHORIA FORMATION IN IDAHO, 1949, PART 2}

By D. F. Davidson, R. A. Smart, H. W. Peirce, and J. D. Weiser

This report concerns work done on

behalf of the U. S. Atomic Energy

Commission and is published with

the permission of the Commission. 


\title{
STRATIGRAPHIC SECTIONS OF THE PHOSPHORIA FORMATION IN IDAHO, 1949, PART 2
}

\author{
By D. F. Davidson, R. A. Smart, H. W. Peirce, and J. D. Weiser
}

CONTENTS

\begin{tabular}{|c|c|c|c|c|}
\hline & Lot no. & Page & Lot no. & Page \\
\hline Introduction. $\ldots \ldots \ldots \ldots \ldots \ldots \ldots$ & & 1 & \multicolumn{2}{|l|}{ Tables of stratigraphic } \\
\hline Acknowledgments. . . . . . . . . . . & & 1 & \multicolumn{2}{|l|}{ sections-Continued } \\
\hline Stratigraphy of the Phosphoria & & & \multirow[t]{2}{*}{ Rocky Canyon. . . . . . . . . . . . . 1304} & 9 \\
\hline formation in southeastern Idaho.... & & 4 & & 13 \\
\hline Stratigraphic sections. . . . . . . . . & & 4 & Gravel Creek Divide. . . . . . . . . 1308 & 16 \\
\hline Selected bibliography..$\ldots \ldots \ldots \ldots \ldots$ & & 4 & Corral Creek.............. 1315 & 19 \\
\hline Tables of stratigraphic & & & Henry.................. 1309 & 20 \\
\hline sections & & & Enoch Valley . . . . . . . . . . . 1314 & 24 \\
\hline Reservoir Mountain. .......... & 1313 & 5 & Ballard trench............. 1316 & 28 \\
\hline \multicolumn{5}{|c|}{ ILLUSTRATIONS } \\
\hline
\end{tabular}

\section{INTRODUCTION}

The U. S. Geological Survey has recently measured and sampled the Phosphoria formation at many localities in Idaho and other western states. These data will not be fully synthesized and analyzed for several years, but segments of the data, accompanied by little or no interpretation, are published as preliminary reports as they are assembled. This is the fifth report of data containing abstracts of sections measured in southea.stern Idaho (fig. 1); it includes about half the data gathered in Idaho in 1949. The field and laboratory procedures adopted in these investigations are described rather fully in a previous report (McKelvey and others, 1953a).

Many people have taken part in this investigation, which was organized and supervised by V. E. McKelvey. F. J. Anderson, A. L. Bush, R. S. Jones, K. B. Krauskopf, K. Lutz, M. E.' Thompson, R. G. Waring, and M. A. Warner participated in the description of strata and the collection of samples referred to in this report. T. K. Rigby assisted in the preparation of trenches and the collection, crushing, and splitting of samples in the field. The laboratory preparation of samples for chemical analysis was done in Denver, Colo., under the direction of W. P. Huleatt.

The $\mathrm{P}_{2} \mathrm{O}_{5}$ and acid-insoluble analyses were made for the Survey by the U. S. Bureau of Mines at the Northwest Electrodevelopment Laboratory, Albany, Oreg., under the direction of S. M. Shelton and M. L. Wright.
The $\mathrm{Al}_{2} \mathrm{O}_{3}, \mathrm{Fe}_{2} \mathrm{O}_{3}$, and loss-on-ignition analyses were made in the Trace Elements Section laboratory of the Survey in Washington, D. C., under the direction of J. C. Rabbitt, by chemists H. Alberty, I. Barlow, A. Caemmerer, N. Guttag, C. Hoy, J. J. Rowe, and W. P. Tucker, and the spectrographic analyses were made in this laboratory by C. L. Waring and $\mathrm{H}$. Worthing.

The data were compiled largely by K. S. Bergman under the supervision of R. W. Swanson. Organization of the tabular data was by Anita Wise.

\section{ACKNOWLEDGMENTS}

Special thanks are due W. W. Rubey, J. Steele Williams, and A. E. Weissenborn who have given much advice in planning and organizing the field program. The cost of the field and laboratory investigations has been partly borne by the Division of Raw Materials of the Atomic Energy Commission.

It is a pleasure to acknowledge the fine cooperation extended to the field parties by the local residents, property owners, and operating phosphate companies, who furnished information and services and gave access to property. A. J. Winters, Superintendent of the Montpelier schools; E. M. Norris, C. T. Russell, and L. E. Traeger of the Anaconda Copper Mining Co.; D. L. King of the San Francisco Chemical Co.; and G. A. $\mathrm{McHugh}$ and H. B. Fowler of the Simplot Fertilizer Co. have been especially helpful in this connection. 


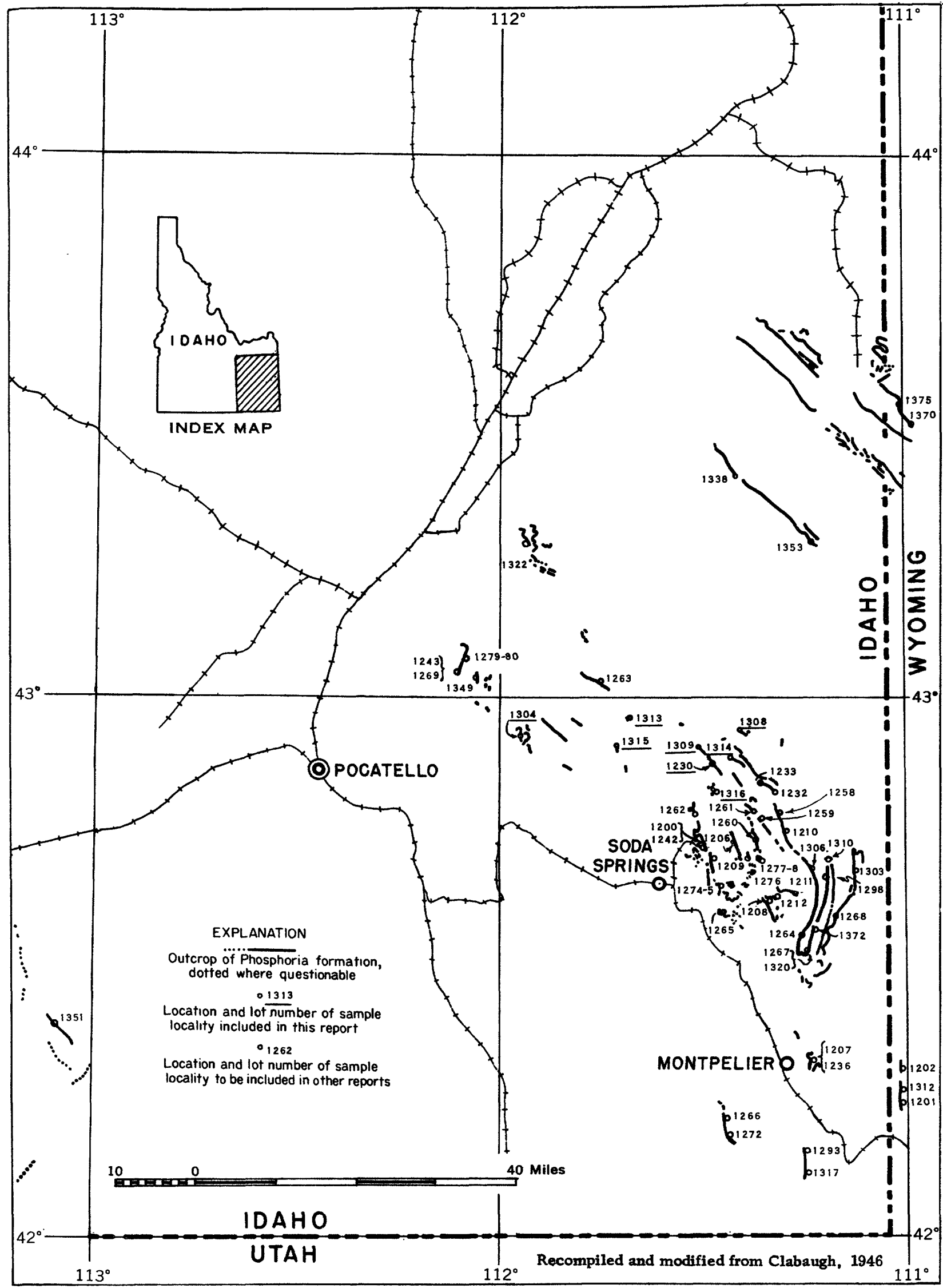

Figure 1. - Outcrops of the Phosphoria formation in Idaho and localities sampled. 


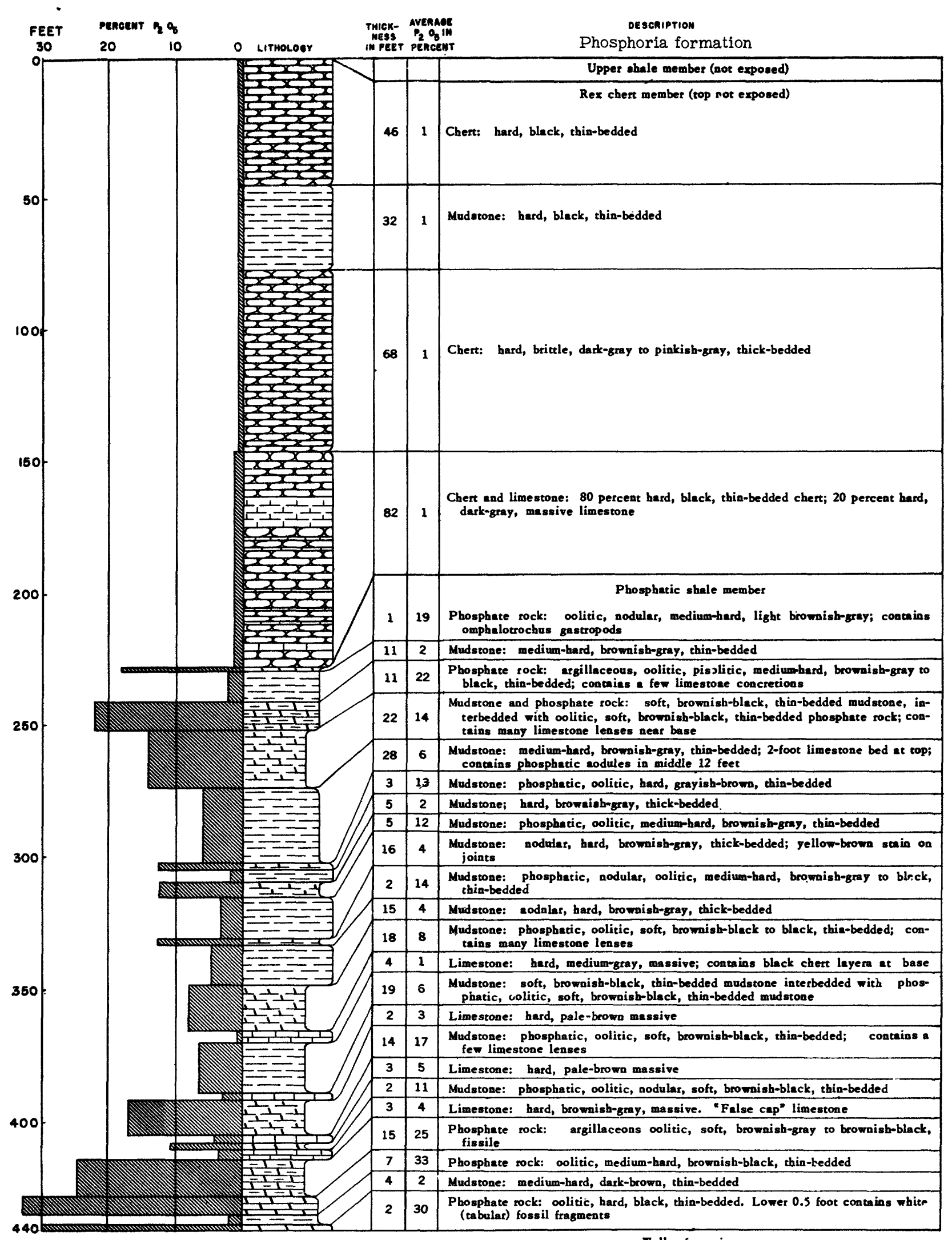

Vells formation

Figure 2. -Generalized section of the Phosphoria formation at Trail Canyon, Idaho, lot no. 1206. 


\section{STRATIGRAPHY OF THE PHOSPHORIA FORMATION IN SOUTHEASTERN IDAHO}

At its type locality in southeastern Idaho (Richards and Mansfield, 1912), the Phosphoria formation consists of a lower member, the phosphatic shale, about 180 feet thick and an upper member, the Rex chert, about 240 feet thick; another member, a thinbedded cherty mudstone 15 to 75 feet thick, overlies the Rex chert member in most of southeastern Idaho and western Wyoming, although it is not well defined at the type locality.

The Phosphoria formation overlies the Pennsylvanian Wells formation and underlies the Triassic Dinwoody formation. The upper 50 to 75 feet of the Wells formation consists of gray fossiliferous cherty limestone that contains some thin phosphatic layers. It may be the correlative the lowermost member (A member) of the Phosphoria formation in Montana and the lower limestone member of the Park City formation in Utah (McKelvey, 1949).

In southeastern Idaho most of the phosphatic beds are in the phosphatic shale member, and it is on this member that most of our studies have been focused. It consists of many thin layers, some of which persist over the whole area. They may be grouped into several broad units, as yet unnamed, as shown in figure 2 .

\section{STRATIGRAPHIC SECTIONS}

Abstracts of stratigraphic sections measured at seven localities, and the available analytical data, are presented in the following pages. Their locations, as well as the locations of those reported previously (McKelvey and others, 1953a and b; O'Malley and others, 1953; and Sheldon and others, 1953), and of others to be reported later, are shown in figure 1 .

The semiquantitative spectrographic analyses are based upon comparison with a standard plate representing known quantities of the elements tested for and made at the same exposure. Greater sensitivities for many elements can be obtained by additional exposure. The standard sensitivities for the elements noted in the report are listed below:

Spectrographic sensitivities

\begin{tabular}{|c|c|c|c|c|c|c|c|}
\hline & Percent & & Percent & & Percent & & Percent \\
\hline $\mathrm{Al}-\cdots---\infty-\infty$ & 0.001 & Gd-n-n-n-n & 0.0 .1 & Nd- - - - - - - & 0.01 & $\mathrm{Na}----------$ & ${ }^{*} 0.1$ \\
\hline 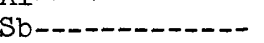 & .01 & $\mathrm{Ga}-\mathrm{C}$ & .01 & $\mathrm{Ni}-\mathrm{NO}-\mathrm{-n}$ & .01 & Sr-- -------- & .01 \\
\hline As-n-n-n-n- & .1 & $\mathrm{Ge}$ & .001 & $\mathrm{Nb}-\cdots-1--\cdots$ & .01 & 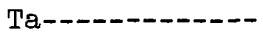 & .1 \\
\hline $\mathrm{Ba}-1-1--1$ & .0001 & $\mathrm{Au}--\cdots$ & .01 & Os_-n & .1 & Ten-n-n-- & .1 \\
\hline 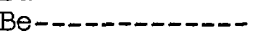 & .0001 & Hf-- - - - - - & .1 & $\mathrm{Pd}--\ldots-1--$ & .01 & $\mathrm{~Tb}------\cdots$ & .01 \\
\hline $\mathrm{Bi}---1---1---$ & .001 & Ho- & .01 & P-- - - & .1 & Tl-- - - - - - & .1 \\
\hline 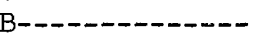 & .001 & In- & .001 & Pt-n- & .01 & Th--n---n--n- & .1 \\
\hline 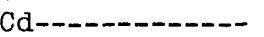 & .01 & Ir-n- & .1 & $\mathrm{~K}-\cdots-1---1-$ & ${ }^{*} 1.0$ & $\operatorname{Tm}------n-\cdots$ & .01 \\
\hline------ & .001 & $\mathrm{Fe}-\ldots$ & .001 & Pr-- & .01 & Sn-- - - - - - - - & .01 \\
\hline------ & .1 & La-- - - - - - - & .01 & Re--n- & .1 & Tin--n---n- & .001 \\
\hline$---\infty$ & $* 1.0$ & $\mathrm{~Pb}---1---1--$ & .01 & 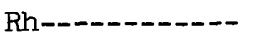 & .01 & 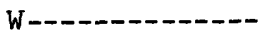 & .1 \\
\hline$-----\infty$ & .001 & Li-n- & $* .1$ & $\mathrm{Rb}-\ldots$ & 10.0 & 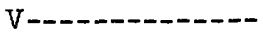 & .01 \\
\hline Co---n--n- & .01 & Lu-n--n--n-- & .01 & Ru--_-_-_--- & .01 & Yb-- - & .0001 \\
\hline----- & .0001 & Mg--10-1- & .0001 & Sm--n-- - - - & .1 & Y-n-n-n- & .001 \\
\hline Dy-- - - - - - - - & .01 & $\mathrm{Mn}-\cdots-\cdots$ & .001 & Sc--n-- & .001 & Znn-n-n-n & .01 \\
\hline---- & .01 & 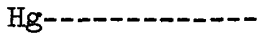 & .1 & ------ & .0001 & Zrr--n-n-n-n & .001 \\
\hline----- & .01 & Mo-- & .001 & $\mathrm{Ag}-1-\cdots-\cdots$ & .0001 & & \\
\hline
\end{tabular}

${ }^{*} \mathrm{~A}$ greater sensitivity may be obtained by additional exposures.

\section{SELECTED BIBLIOGRAPHY}

Clabaugh, P. S., 1946, Permian phosphate deposits of Montana, Idaho, Wyoming, and Utah: U. S. Geol. Survey, Strategic Minerals Inv. Prelim. Map 3-198.

McKelvey, V. E., 1949, Geological studies of the western phosphate field: Am. Inst. Min. Met. Eng. Mining Trans., v. 184, p. 270-279.

McKelvey, V. E., Armstrong, F. C., Gulbrandsen, R. A., and Campbell, R. M., 1953b, Stratigraphic sections of the Phosphoria formation in Idaho, 1947-48, pt. 2: U. S. Geol. Survey Circ. 301.

McKelvey, V. E., Davidson, D. F., O'Malley, F. W., and Smith, L. E., 1953a, Stratigraphic sections of the Phosphoria formation in Idaho, 1947-48, pt. 1: U. S. Geol. Survey Circ. 208.
O'Malley, F. W., Davidson, D. F., Hoppin, R. A., and Sheldon, R. P., 1953, Stratigraphic sections of the Phosphoria formation in Idaho, 1947-48, pt. 3: U. S. Geol. Survey Circ. 262.

Richards, R. W., and Mansfield, G. R., 1912, The Bannock overthrust: a major fault in southeastern Idaho and northeastern Utah: Jour. Geology, v. 20, p. 684 .

Sheldon, R. P., Warner, M. A., Thompson, M. E., and Peirce, H. W., 1953, Stratigraphic sections of the Phosphoria formation in Idaho, 1949, pt. 1: U. S. Geol. Survey Circ. 304. 


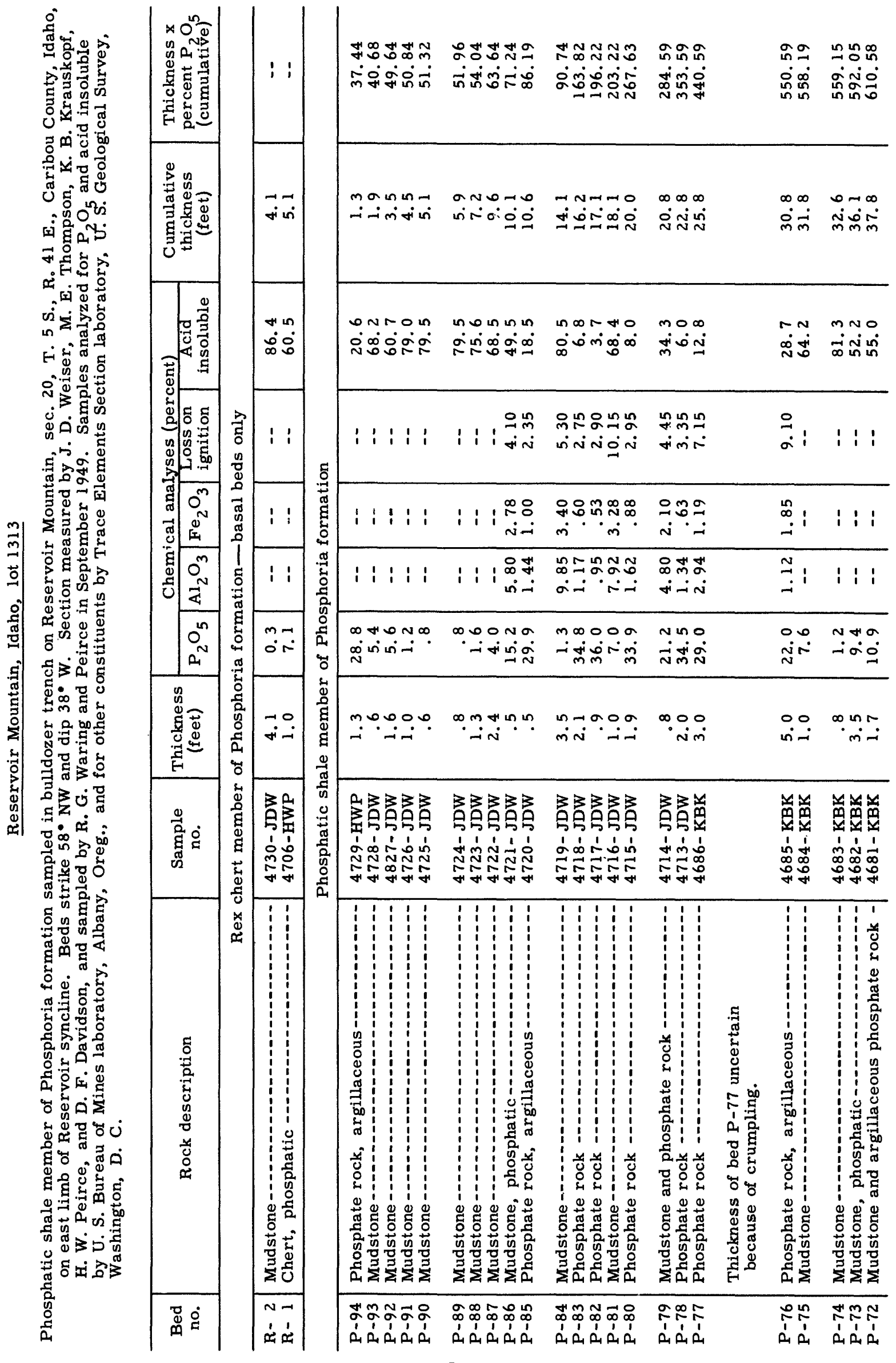




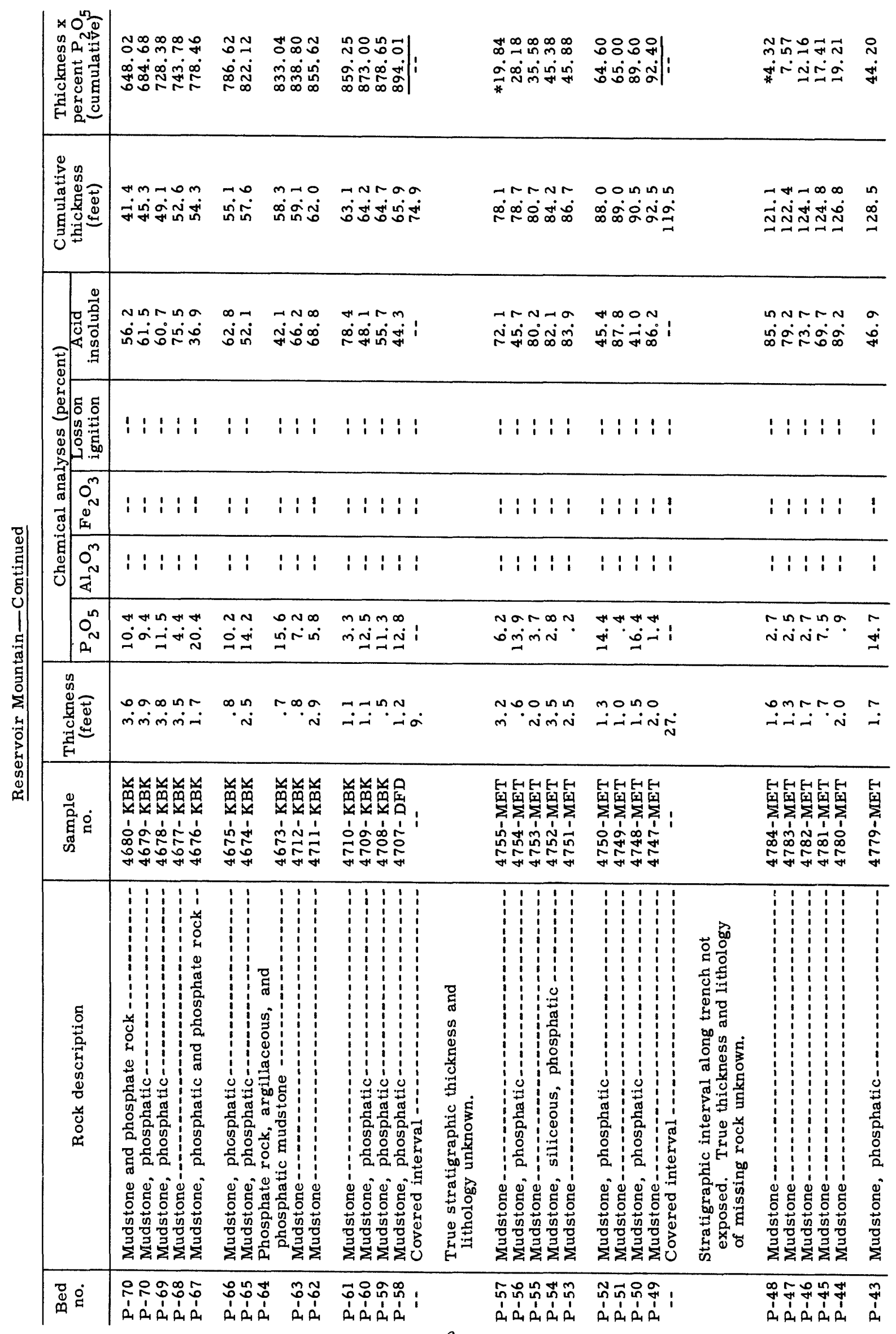




\begin{tabular}{|c|c|c|c|c|c|c|c|c|}
\hline 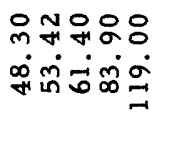 & 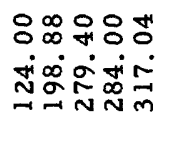 & 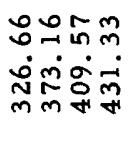 & \begin{tabular}{l}
$\tilde{0}$ \\
$\dot{0}$ \\
$\vdots$ \\
\multirow{H}{*}{}
\end{tabular} & 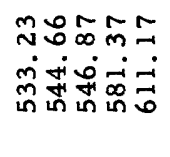 & 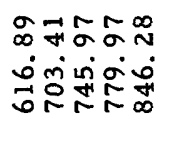 & $\begin{array}{l}N \alpha \\
\sigma \alpha \\
\therefore \circ \\
\infty \\
\infty\end{array}$ & 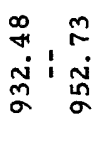 & 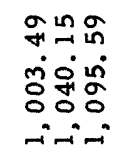 \\
\hline
\end{tabular}

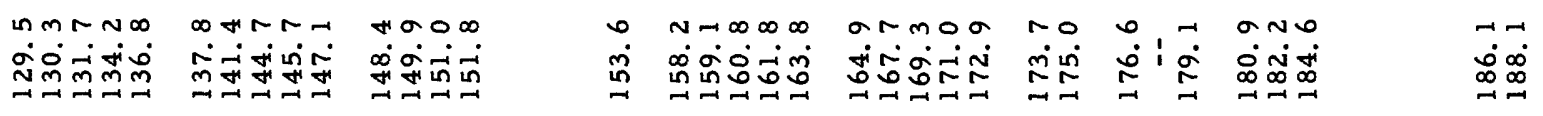

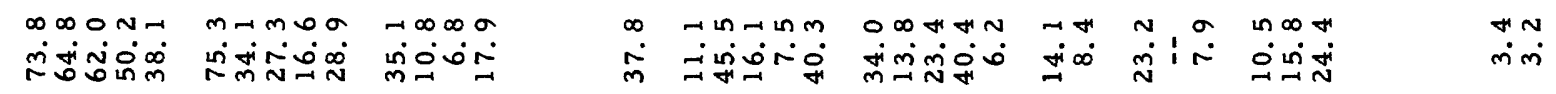

\begin{tabular}{|c|c|c|c|c|c|c|c|c|c|c|c|}
\hline $\begin{array}{lllll}1 & 1 & 1 & 1 & 1\end{array}$ & 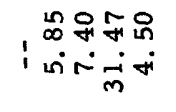 & 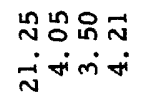 & & $\begin{array}{l}\alpha \\
\dot{\pi}\end{array}$ & 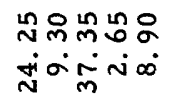 & 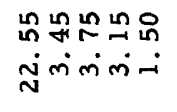 & $\begin{array}{l}\text { : } \\
\text { io }\end{array}$ & $\begin{array}{ll}n & 0 \\
m & \infty \\
& 0 \\
& 0\end{array}$ & $\begin{array}{l}\text { 눙ㅇㅇㅇ } \\
\text { imió }\end{array}$ & & 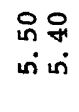 \\
\hline 1: $1: 1: 1$ & 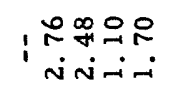 & 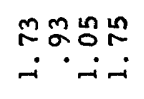 & & $\begin{array}{l}\infty \\
\infty \\
\ddot{-}\end{array}$ & 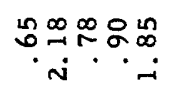 & 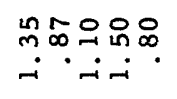 & 倇 & $\begin{array}{lll}n & \text { in } \\
0 & 0 & 0 \\
-1 & 0\end{array}$ & $\stackrel{\sim}{\sim} \tilde{m}$ & & mె \\
\hline $1:$ & 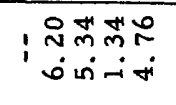 & 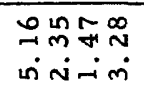 & & $\stackrel{\circ}{\sim}$ & 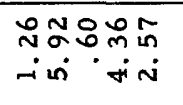 & 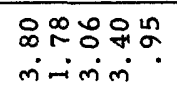 & $\begin{array}{l}\overrightarrow{0}= \\
\dot{N}=\end{array}$ & $\tilde{m}: \stackrel{p}{m}$ & 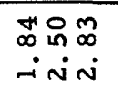 & & $\stackrel{0}{\sim 5}$ \\
\hline $\begin{array}{l}\text { Hino in } \\
\text { Hiong }\end{array}$ & ம் & rom & & $\stackrel{m}{2}$ & 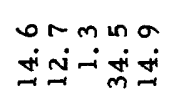 & Nö & $\begin{array}{l}\infty 0 \\
\dot{0} \dot{m}\end{array}$ & $\ddot{\infty}$ & NNm & & $\dot{0} \infty$ \\
\hline ن̊ & :0mö & 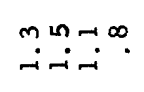 & & $\stackrel{\infty}{-}$ & 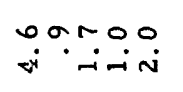 & تُ & $\stackrel{\infty}{\because}$. & iْnn & 舫. & & in \\
\hline 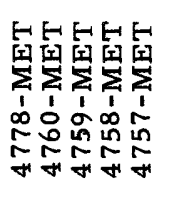 & 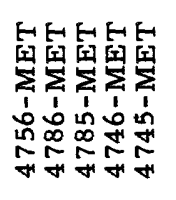 & 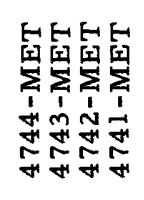 & & 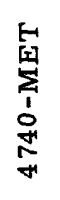 & 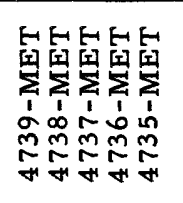 & 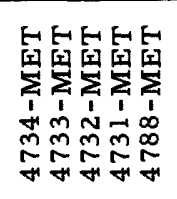 & 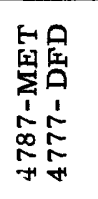 & 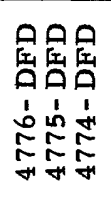 & 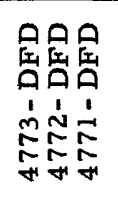 & & 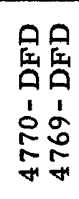 \\
\hline 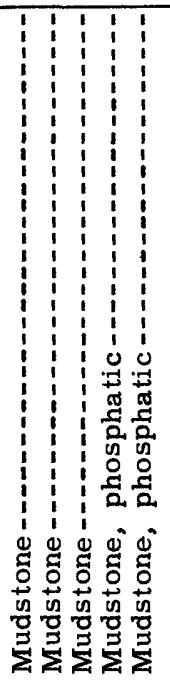 & 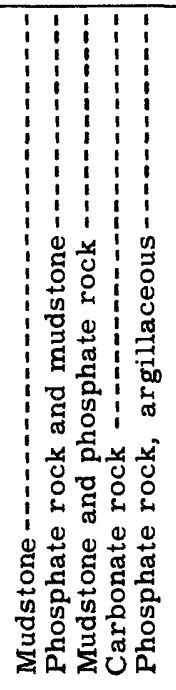 & 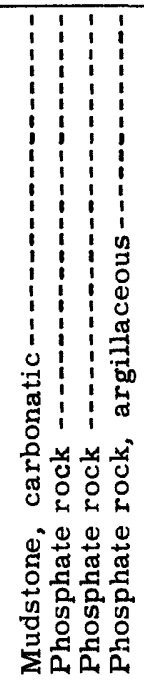 & 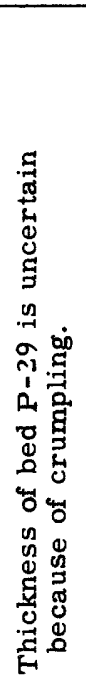 & 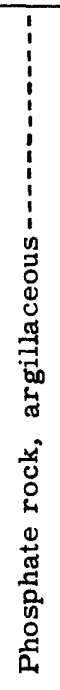 & 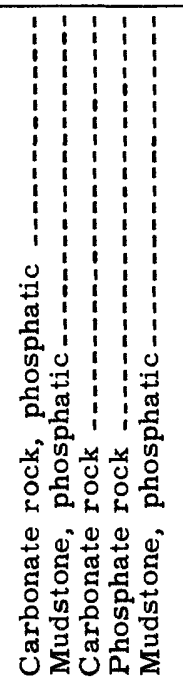 & 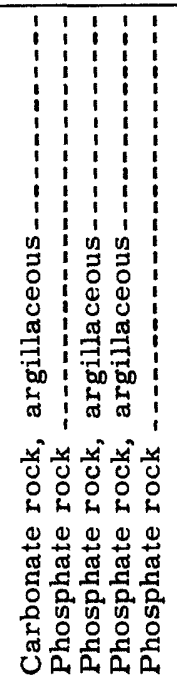 & 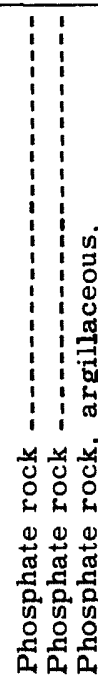 & 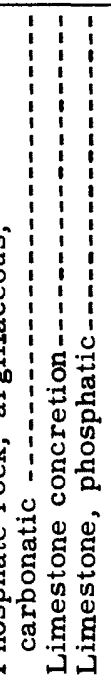 & 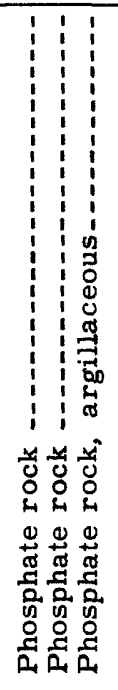 & 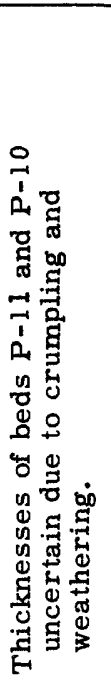 & 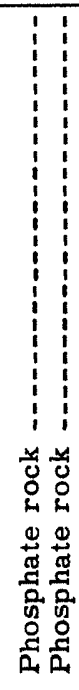 \\
\hline 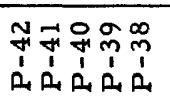 & 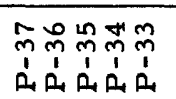 & 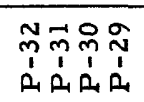 & & $\begin{array}{l}\stackrel{\infty}{N} \\
\vdots \\
n\end{array}$ & 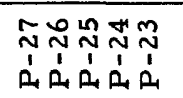 & 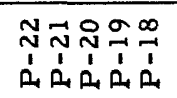 & 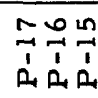 & ì & 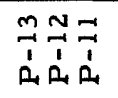 & & $\begin{array}{l}\circ \sigma \\
\dot{1} \\
a_{1}\end{array}$ \\
\hline
\end{tabular}




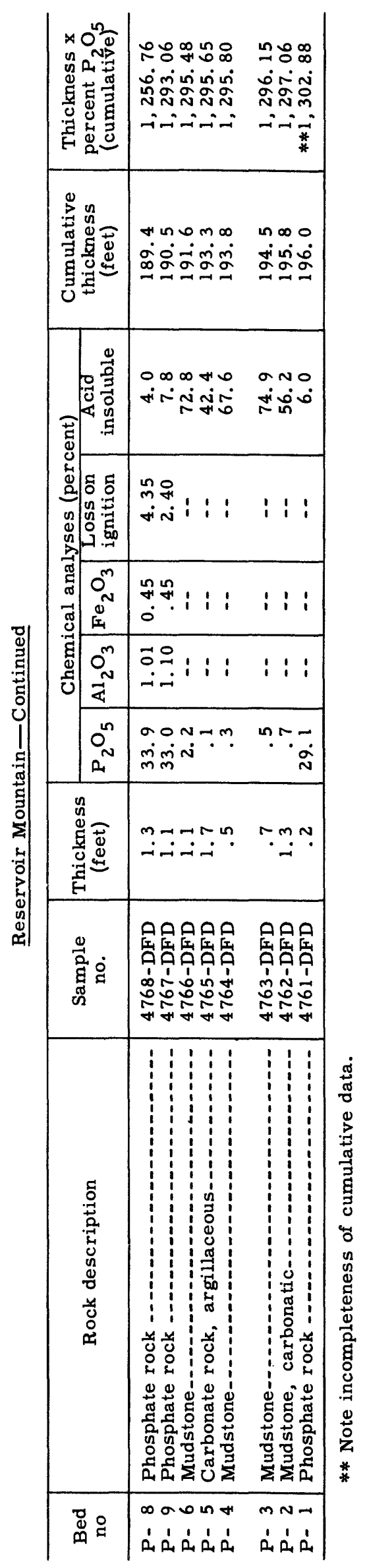




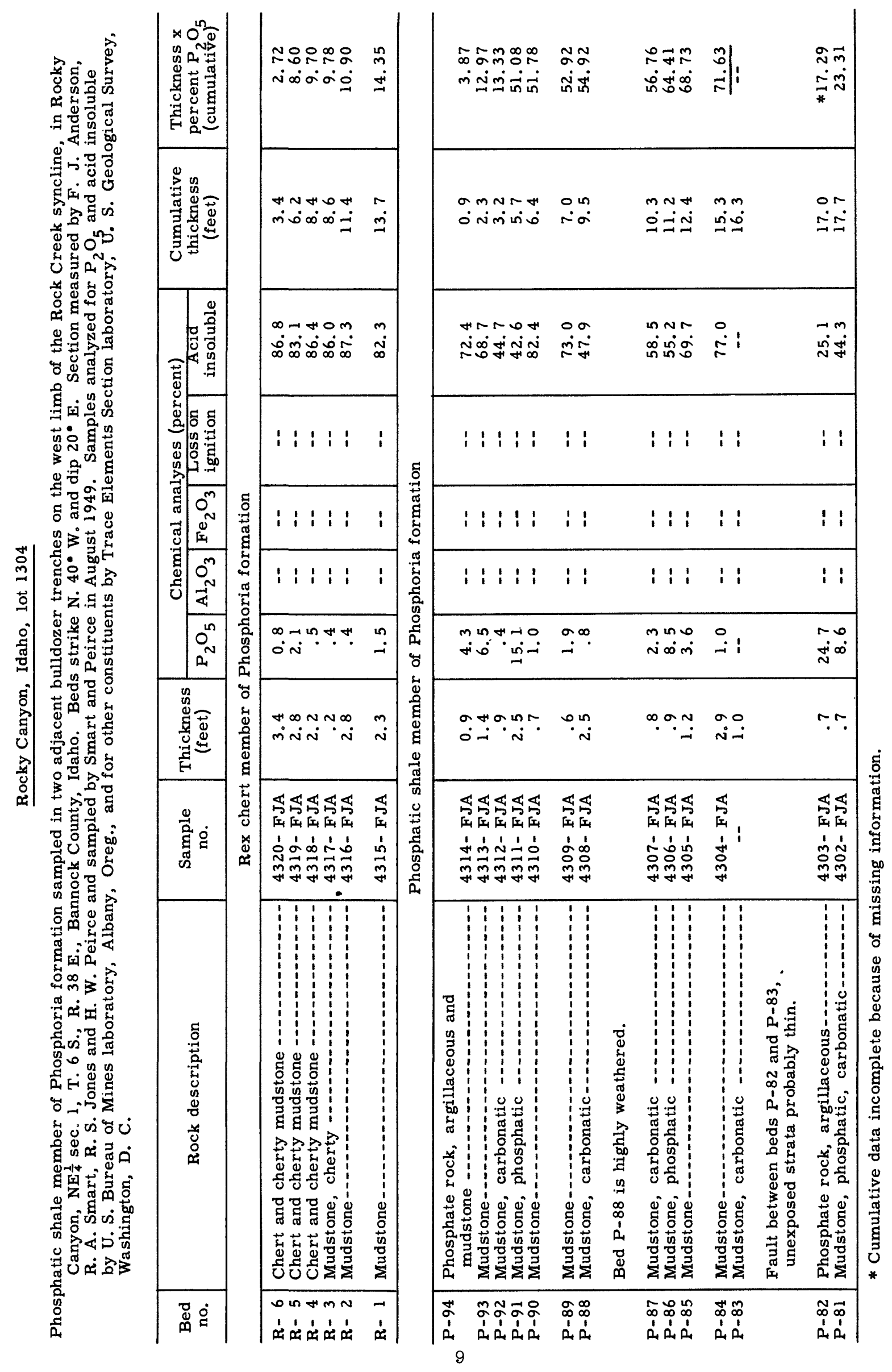




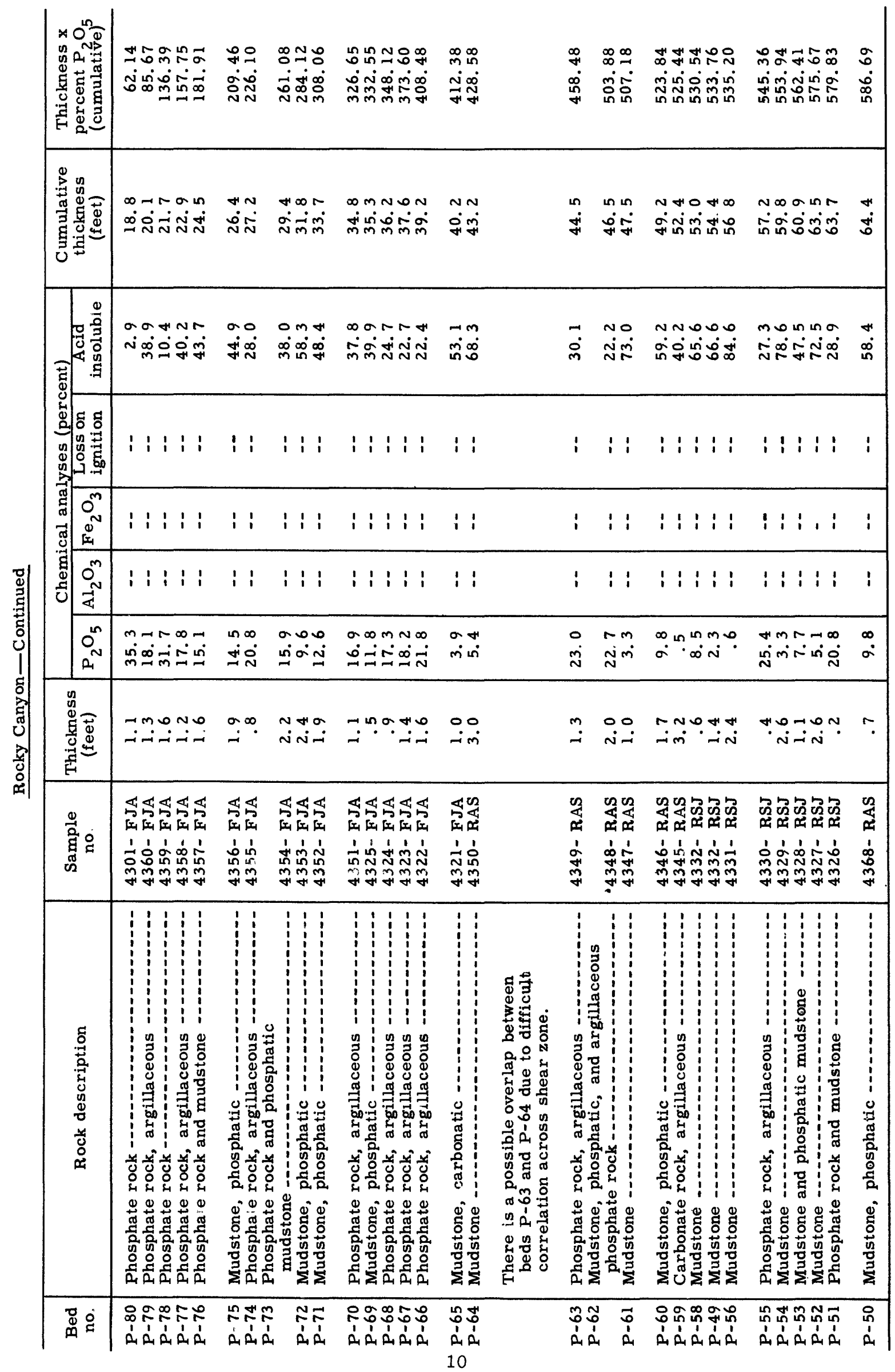




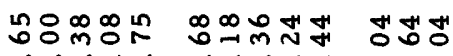

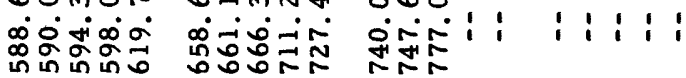

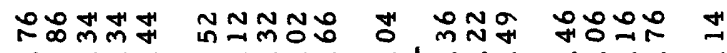

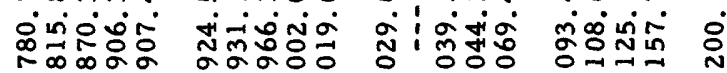

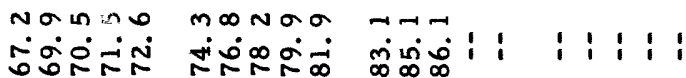

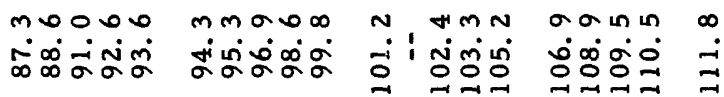

monom ñm
Nmtron

NลNกำ othmn

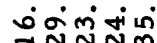

NhoOH TONH N

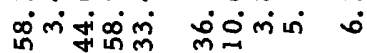

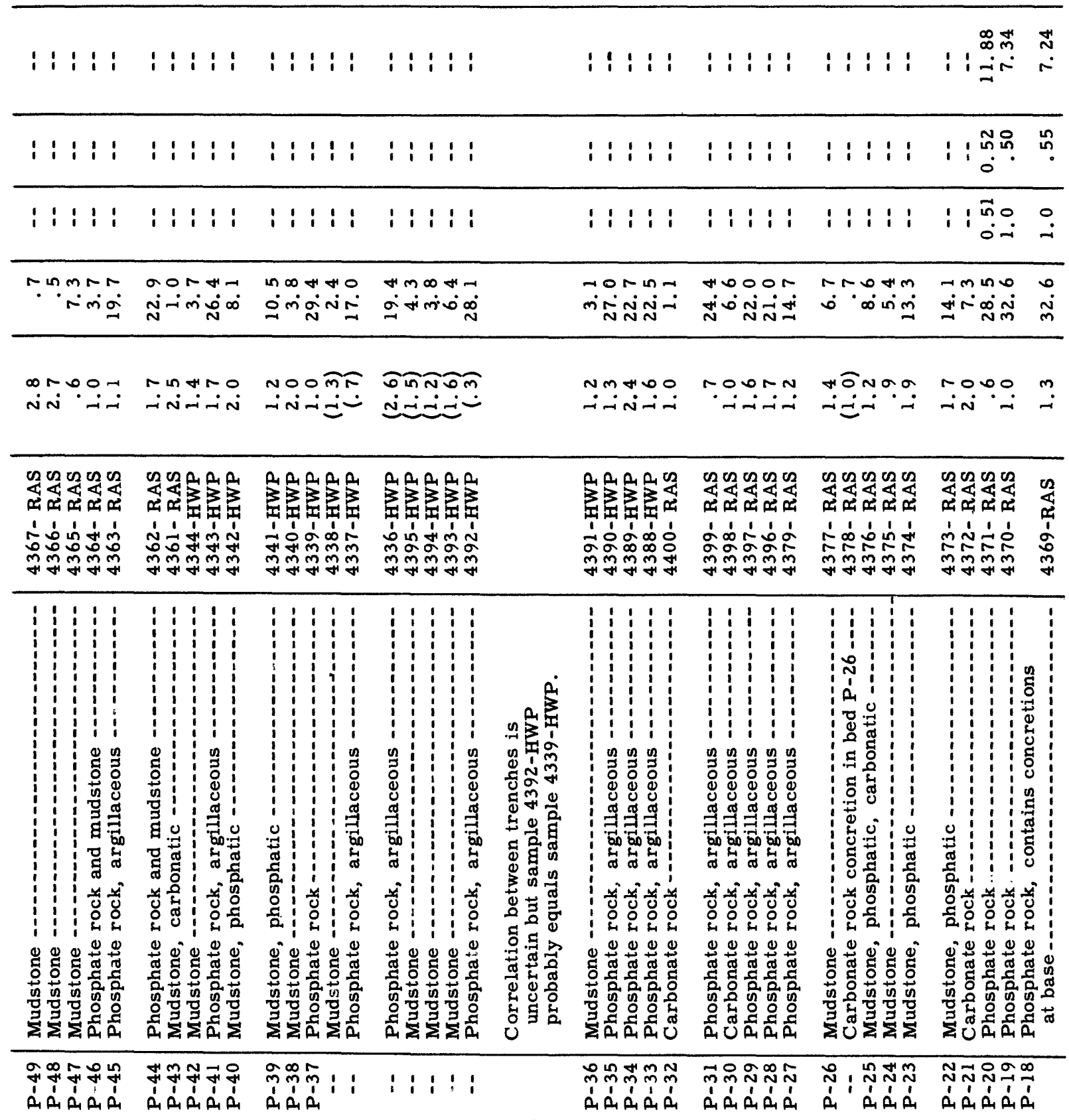




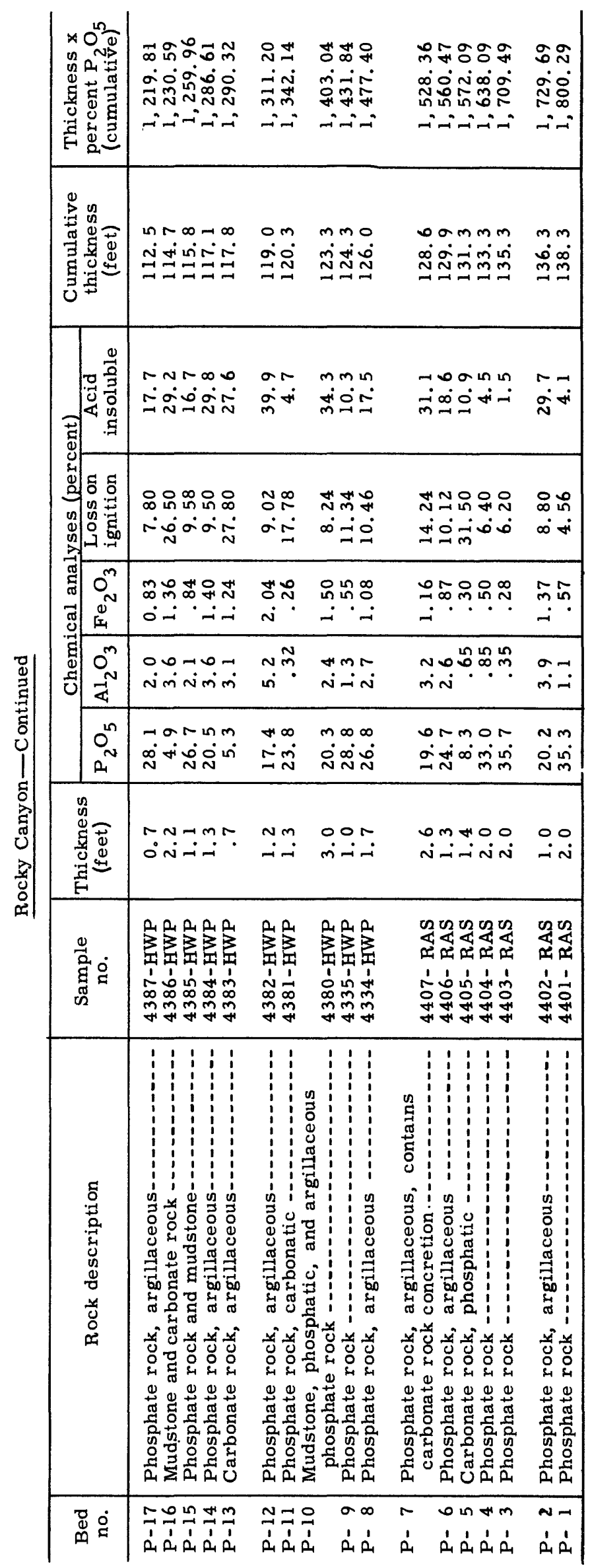




\begin{tabular}{|c|c|c|c|c|c|c|c|c|}
\hline$\hat{N}$ & 四质回四四 回 & 뵈되 되되 뙤 & 되보되 되 & 回质质质回 & 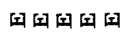 & 国国国国国 & 质压质国四 & 뵈되되뵈보 \\
\hline & 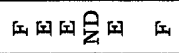 & 最的的四四 & 되을되되디 & 合合的合的 & 되뵈터보도 & 뵈되뵈뵈되 되 & 되되되뵈비 & 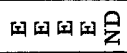 \\
\hline & 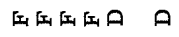 & 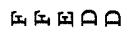 & คロロ日十 & 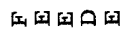 & 뵈뵈되더비 & 뵈되되뵈되 & 国の国国国 & 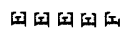 \\
\hline & 0000 四 & 比田质 & 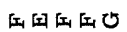 & 四田四四 & 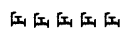 & 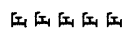 & 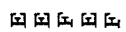 & 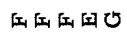 \\
\hline
\end{tabular}

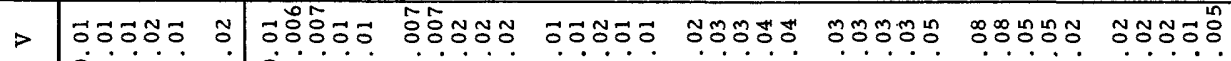

\begin{tabular}{|c|c|c|c|c|c|c|c|c|}
\hline r & คの日电回 回 & 뵈뵈 뵈시 & 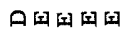 & 口回国的 & คคคคค & คคคคค & 뵈뵈서요 & ค四のค回 \\
\hline
\end{tabular}

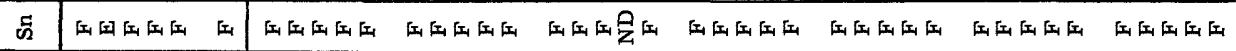

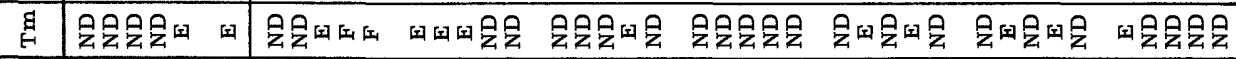

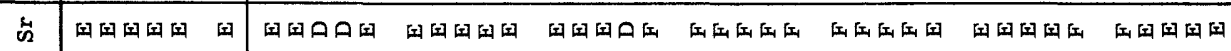

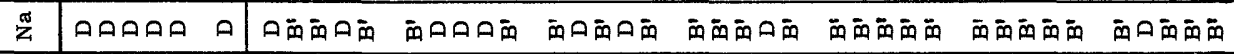

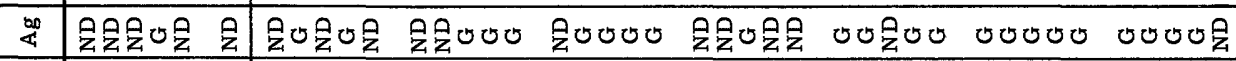

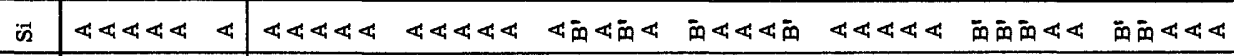

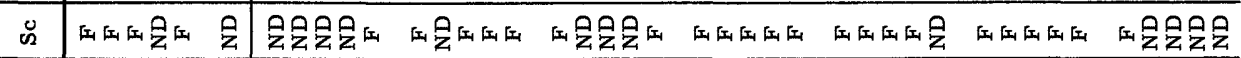

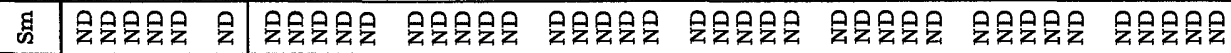

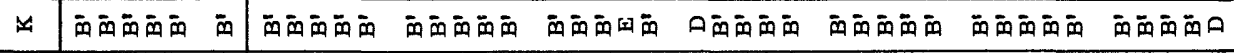

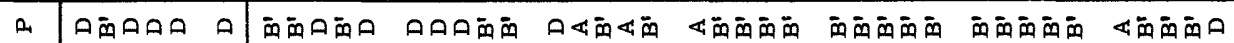

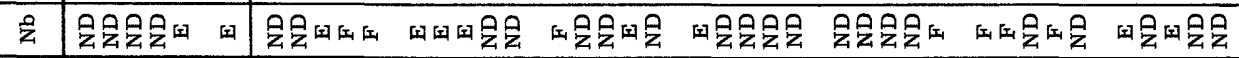

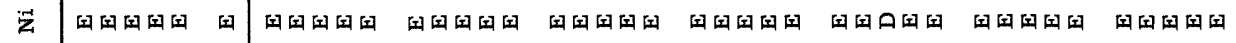
艺

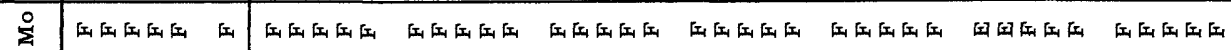

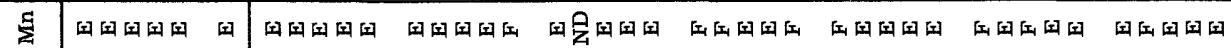

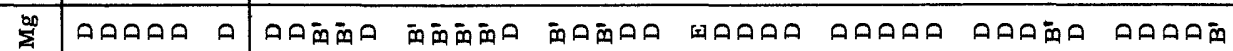

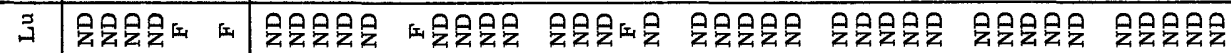

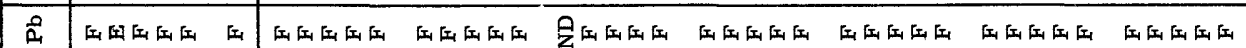

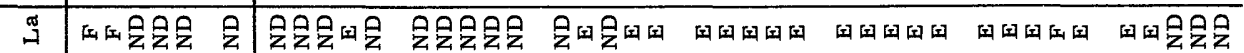

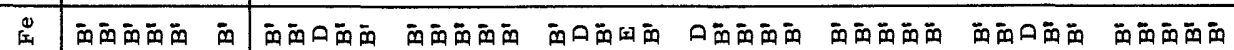

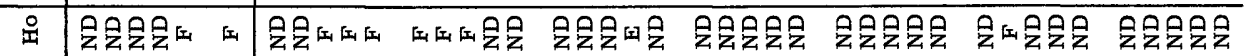

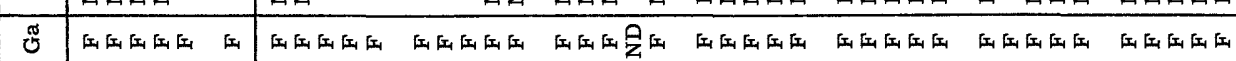
ర

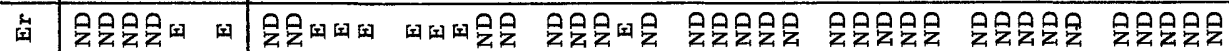

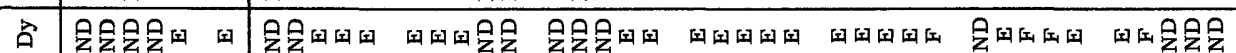

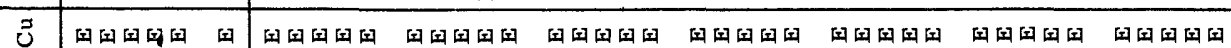

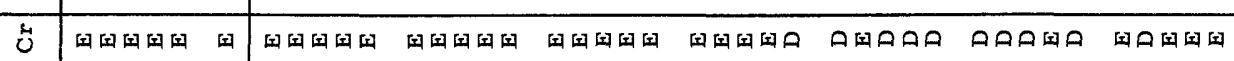

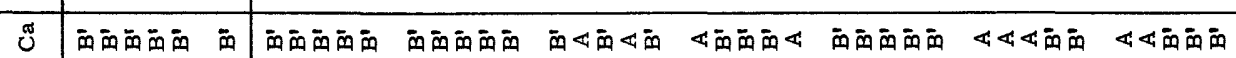

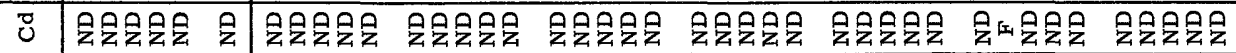

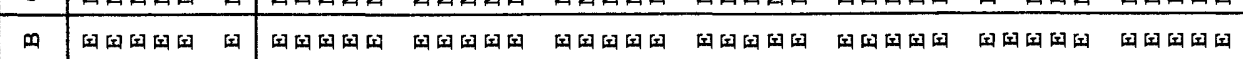
芩

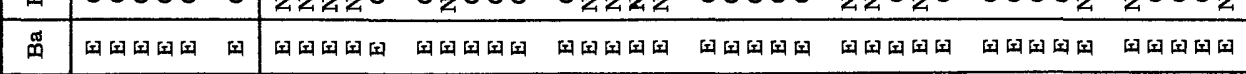

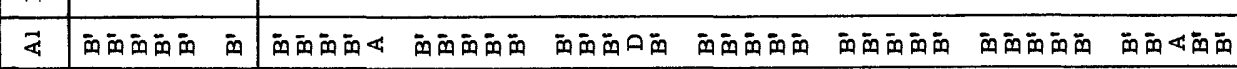

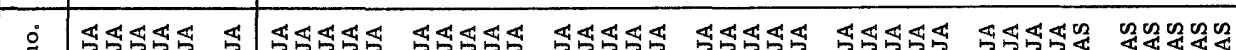

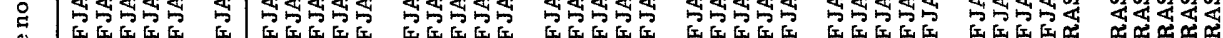

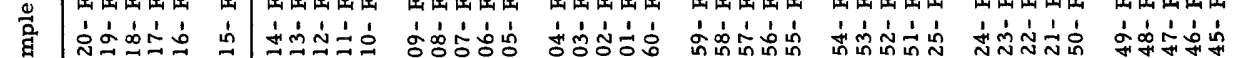

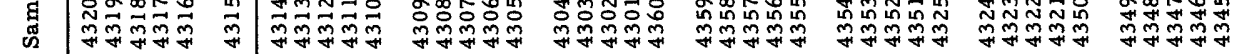

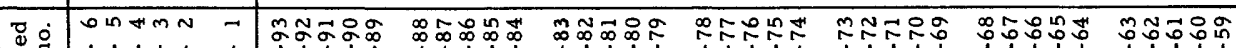

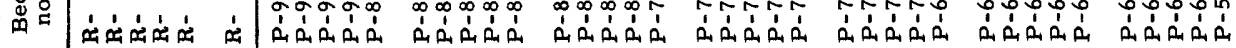




\begin{tabular}{|c|c|c|c|c|c|c|c|c|c|c|c|}
\hline & & & & & & & 费国 & & & & \\
\hline & 的的会会会 & 国 & 9 & $=$ & 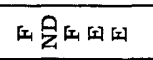 & & 뙤 & & & & \\
\hline & F & 国 & a & 国 & 뵈뵈버비 & 뵈뵈되되되 & 活国 & & & & \\
\hline$x$ & 뵈되되응 & 4 & E & 的的的压 & 的国的质 & 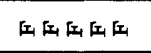 & $0 \sqrt{4}$ & & 㝳 & 屈 & \\
\hline$>$ & $\begin{array}{l}0 \\
\vdots\end{array}$ & 0.80 & $D_{-4 m}$ & Nลัด- & 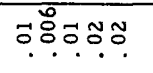 & ตํํำ & ตุ & & & & ํํㅇ \\
\hline & 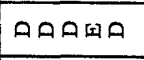 & Q.90 & 0 & 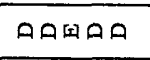 & $\triangle ค ค ค ค$ & & & & 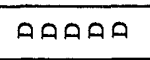 & & \\
\hline- & 压的会国 & 庄的 & & & & & 4 & & & & \\
\hline$\stackrel{g}{E}$ & 뵈되 뙤을 & 县, 되되 & $\overline{2}$ & 是会 & 最是是 & 全会会的 & 的会会 & 国国 & & & \\
\hline in & 뙤되 9 미 & 国、的的 & 和的的压 & 되뵈뵈되 & 되 & 応的的 & 的的的匠 & If & & & \\
\hline$\pi^{\pi / 2}$ & 向向口向 & 畐 & & & 向口向 & 西向 & ā & & & & м̄ \\
\hline 8 & 宝全金安 & 5 & & 5 & b & 000 & 50 & & & & $\pi$ \\
\hline$\ddot{n}$ & 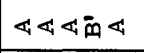 & 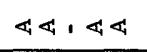 & & 4 & & 4 & & & & & 商 \\
\hline in & 是会的是 & 压的 & 毛的 & 题呈 & 量 & 的的是 & $\overline{\mathrm{O}}$ & 最是 & 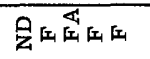 & & 号会 \\
\hline 量 & 县的会 & 只、公㫕 & 是 & 会 & 会 & 会会兄会会 & & & & 县会 & 县会 \\
\hline 4 & 向的向向 & & & 可畐的向 & $\overline{\bar{m}}$ & 向的的亩向 & & $\overline{\text { 同A }}$ & & & $\overline{\mathrm{m}} \overline{\mathrm{x}}$ \\
\hline A & DA & 两口 & & 的 & $\overline{\text { m }}$ & 畐的的畐口 & 口向 & 西口 & 向畐 & & $\overline{\mathrm{m}}$ \\
\hline$\frac{8}{2}$ & 四会的 & 另, 的的 & 回的会号 & 哈会会 & & 的的的的 & 的的 & 政的 & 되 & & \\
\hline$z$ & 뵈되되도 & 1되 । 뵈뵈 & 되퇴되디 & 뵈뵈 되되 & 뙤되되디 & 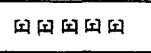 & 되되 & 站 & 되 & 国 & 国 \\
\hline z & 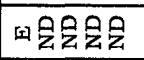 & $\overline{\mathrm{z}}$ & & 最是是 & 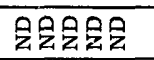 & 全会全会会 & 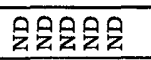 & 会㕕会全会 & 完会会会 & & $z$ \\
\hline$\sum_{\Sigma}^{\circ}$ & 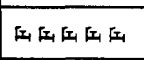 & 所，反的 & 乐原的原 & 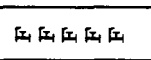 & 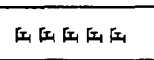 & 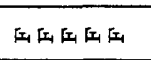 & 质的 & 的的的的的 & 的的的的的 & Fis 压 & 的厌 \\
\hline $\bar{\Sigma}$ & 뵈되도 되 & 成 & 네 똬 & 뵈되 & 푀 & 뵈데 뙤띠 & 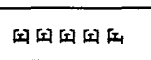 & & 되 & & \\
\hline$\sum_{20}^{20}$ & ๓คคค & $a$ & a & A & $\bar{m}$ & $A D$ & की & $A D$ & $O A$ & $A$ & $a c$ \\
\hline 3 & 会全全呈 & 会 & i⿱ & 完会 & 会会全是 & 是是会会 & $\mathrm{QP}$ & 量会 & 会会 & 20 & 公 \\
\hline$\hat{A}$ & is & & & & & 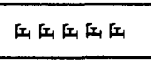 & 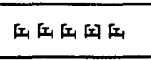 & 质的压的会 & 化质的质 & & \\
\hline$\stackrel{g}{\unlhd}$ & 死的国卧 & & & 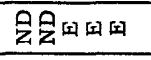 & 会完利的 & 버퇴 떠터 터 & 퇴터 되터 떠 & 뙤되뫼을 & & & \\
\hline 哯 & in & & & 商口西向 & A商而向 & का & 的畐口可 & & & & 中心 \\
\hline 온 & 织 & 岳圷 & 的的公会 & 兊会 & 公会会号 & 応质的合 & Ex I & $x_{1} x_{1}$ & 得会会的 & & 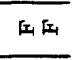 \\
\hline : & 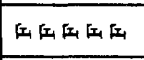 & 4 & 工毛 & 的的的应 & 的的的的 & I & 平庄 & 地是 & 任的的的 & & 45 \\
\hline 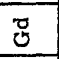 & 뵈뵈비굴 & & & & & & & & & & $\bar{z}$ \\
\hline 夋 & 金金云 & & & & & & 8 & 锭 & & & 完: \\
\hline a & 所的四吴 & 的会, 的的 & 다 다 & 会会旦会 & 全会质 & 会会全全的 & 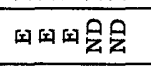 & & 利的质的原 & 的层的层国 & 国 \\
\hline U & 뵈뵈뵈뵈 & 뙤 1 뵈되 & वा国 & 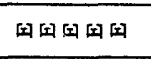 & 뵈 뫼되 되 & 뵈터뵈디 & 国国 & 9 뙤터 퇴디 & |미 & a国 & $\Leftrightarrow$ \\
\hline 0 & 뵈뵈 뙤디 & 0 & & 国 & 四 & (1⿴囗十 & 0 & & & 中国 & 幽 \\
\hline$\pi$ & 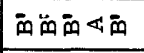 & & & & & & & 4 & & & $\pi$ \\
\hline i & 是㫕会公 & 公公・会会 & & 帘是是会 & 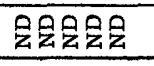 & 是公会品 & & & G4 & & \\
\hline 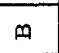 & 되뵈되티 & 뵈되，四国 & 뙤되되되되 & 뵈퇴뙤뵈되 & 뵈되 & 国国的国 & 的所的的国 & 되되되되을 & 国 & & 政 \\
\hline Ф & 0 & & & 5会 & 8000 & 爷最最 & 00 合安会 & & 坛会会 & 会会 & $\frac{1}{2}$ \\
\hline m & 되 되되되되 & 뙤되 约国 & 되되 되뵈 & 되뵈 & 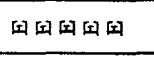 & 国国国国 & 뵈뵈 퇴이 & 되 떠도 & 법뵈뵈 되 & 田国 & 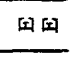 \\
\hline 4 & Q $\varangle \bar{m} \varangle$ & 向・畐 & & 《勾向向 & 向《的向 & 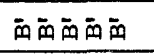 & 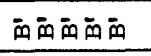 & $Q A$ & & & m \\
\hline $\begin{array}{l}\text { : } \\
\ddot{\alpha}\end{array}$ & 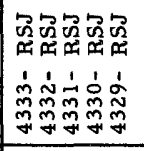 & 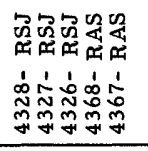 & 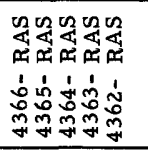 & 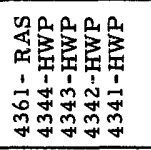 & 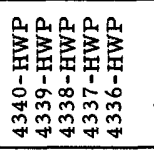 & 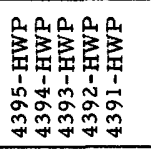 & 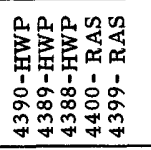 & $\begin{array}{l}2 \\
0 \\
0\end{array}$ & 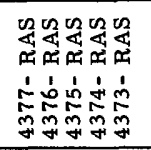 & 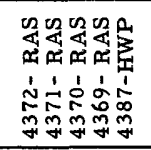 & 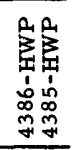 \\
\hline & 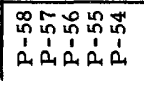 & & & & & & & & & & 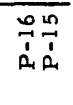 \\
\hline
\end{tabular}




\begin{tabular}{|c|c|c|}
\hline 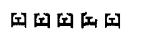 & 所死四四四 & 뙤되되 \\
\hline 四四四匠田 & 뙤더 떠 띠 & 뙤되되 띠 \\
\hline 国国闵国田 & 되 뙤 뙤뵈 되 & 뙤뙤되 \\
\hline 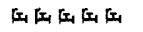 & 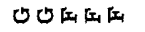 & 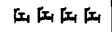 \\
\hline ํํํㅇํํํำ & ๑ำอีอ & ชั่ำㅇํำ \\
\hline คคロ四の & 田的คคค & คคคค \\
\hline 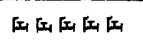 & 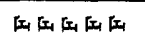 & 的的的合 \\
\hline 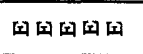 & 公会是会号 & 会会吴吴 \\
\hline 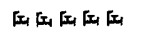 & 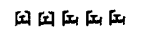 & 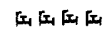 \\
\hline 向口向的的 & 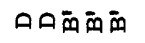 & 的向的商 \\
\hline ט טט טט & טు טטు & 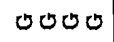 \\
\hline 向的向口的 & 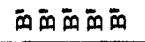 & 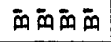 \\
\hline 号是昌最昌 & 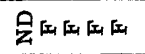 & 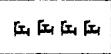 \\
\hline 公是是公呈 & 公是是公会 & 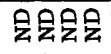 \\
\hline 的向的问 & คค向向的 & $\bar{m} \bar{m} \bar{m} \bar{m} \bar{m}$ \\
\hline 的的的的的 & $\varangle \varangle \bar{n} \bar{n} \bar{m}$ & 的的商向 \\
\hline 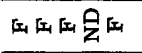 & 会会质应质 & 压压压质 \\
\hline 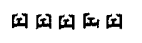 & 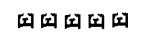 & 红国四四 \\
\hline 是是是是是 & 公公公是呈 & 会昙只只 \\
\hline 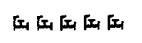 & 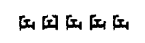 & 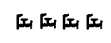 \\
\hline 四四四问问 & 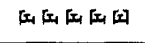 & 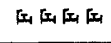 \\
\hline คคคคค & คคคคต̄ & คคคค \\
\hline 公㕕是是会 & 公公公是只 & 公公公会 \\
\hline 的的的号的 & 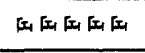 & 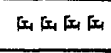 \\
\hline 四四四国垊 & 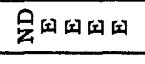 & 国国四国 \\
\hline 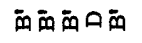 & A舟的可口 & 的の的口 \\
\hline 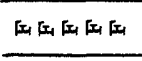 & 公是的的瓜 & 压底厌原 \\
\hline 的的原是的 & 号的的的原 & 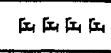 \\
\hline 最会旦是是 & 公公会是是 & 公会会会 \\
\hline 公公公公会 & 公会会公是 & 县是是会 \\
\hline 邱的国会的 & 公公号会会 & 是最最吕 \\
\hline 国四四国四 & 四四四国夙 & 뵈비법 \\
\hline ค目の四口 & 뵈봄되 & 뵈되되되 \\
\hline 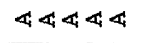 & $\varangle \varangle \ll \ll 4$ & $\varangle \varangle \varangle$ \\
\hline 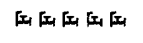 & 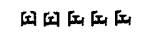 & 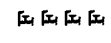 \\
\hline 回出四山四 & 国四四四盾 & 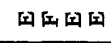 \\
\hline 公是是是兄 & 会口会会是 & 是是是是 \\
\hline 国四国四四 & 国四四四回 & 뙤뵈 뵈보 \\
\hline 向向的口向 & 向向的向口 & 的の的口 \\
\hline 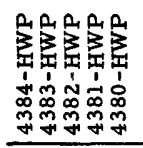 & 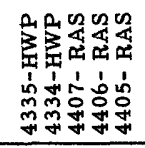 & 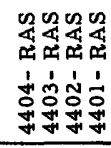 \\
\hline 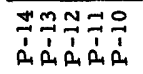 & 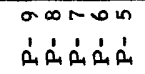 & 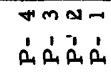 \\
\hline
\end{tabular}




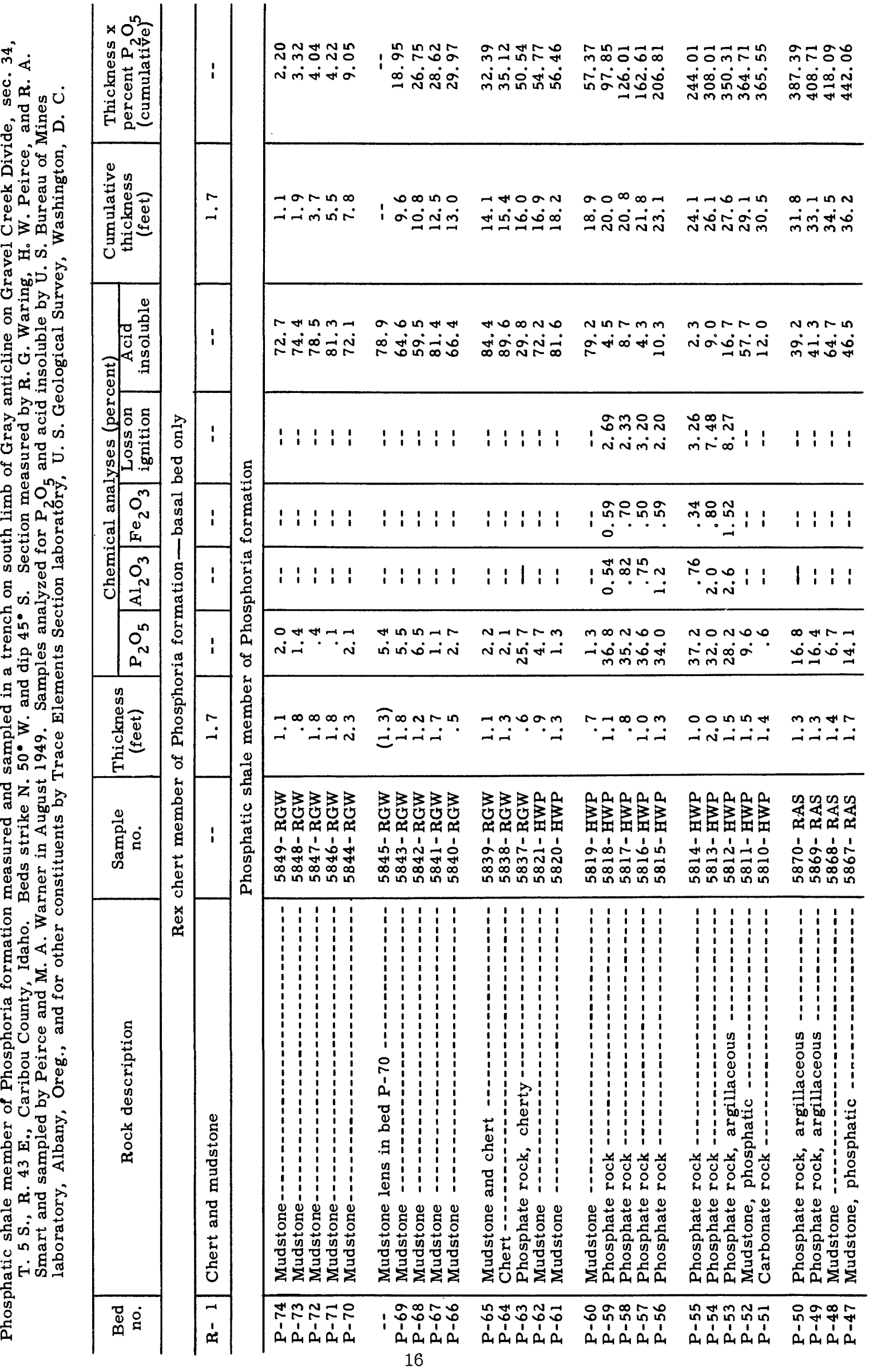




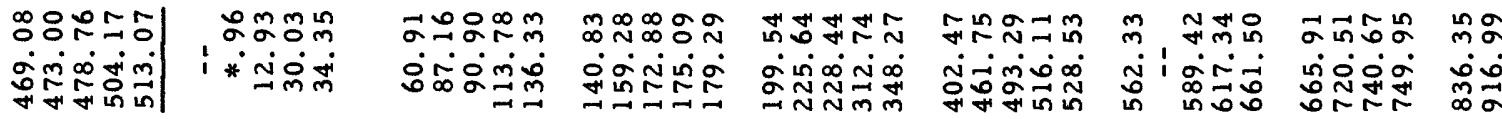

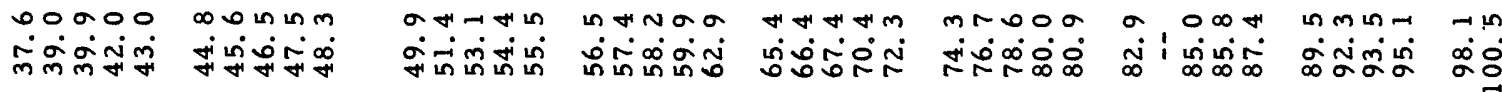

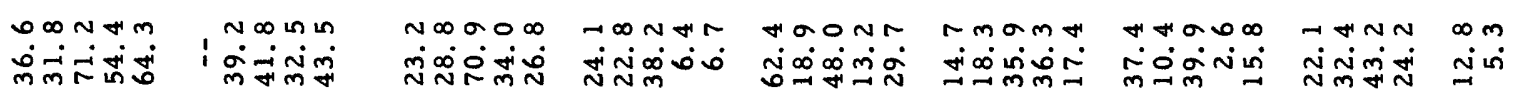



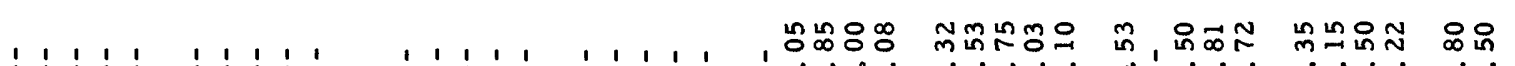

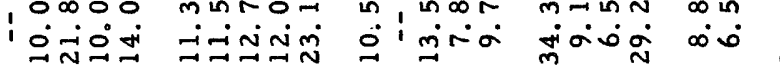

\begin{tabular}{|c|c|c|c|c|c|c|c|c|}
\hline $\begin{array}{lllll}1 & 1 & 1 & 1 & 1\end{array}$ & $\begin{array}{lllll}1 & 1 & 1 & 1 & 1\end{array}$ & $\begin{array}{lllll} & 1 & 1 & 1 & 1\end{array}$ & $\begin{array}{lllll} & 1 & 1 & 1 & 1\end{array}$ & $\begin{array}{l}\overrightarrow{0} \mathrm{~m} \\
\dot{i} \\
\end{array}$ & \begin{tabular}{c}
$\infty: 8$ \\
\hdashline-17
\end{tabular} & 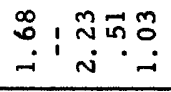 & 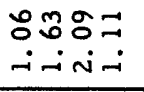 & $\underset{0}{0} \stackrel{\infty}{\stackrel{n}{\circ}}$ \\
\hline i i & 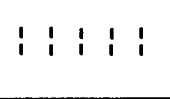 & 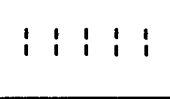 & 1911 & I Nợ & Niñ & ㄱำ & $\begin{array}{l}\infty \infty \sim \sim N \\
\text { imin }\end{array}$ & i̊ \\
\hline 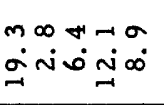 & immin & 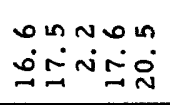 & 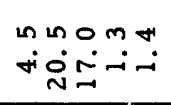 & 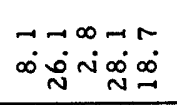 & ت்̃0m & $\begin{array}{l}a \sigma a \\
0 \\
0\end{array}$ & ن & $\begin{array}{l}\infty \\
\infty \\
N \\
N\end{array}$ \\
\hline
\end{tabular}

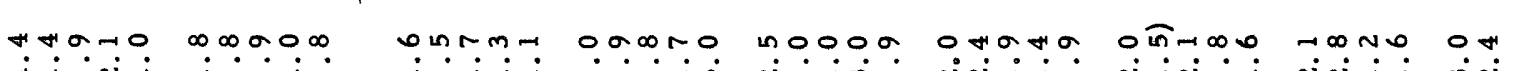

inh-

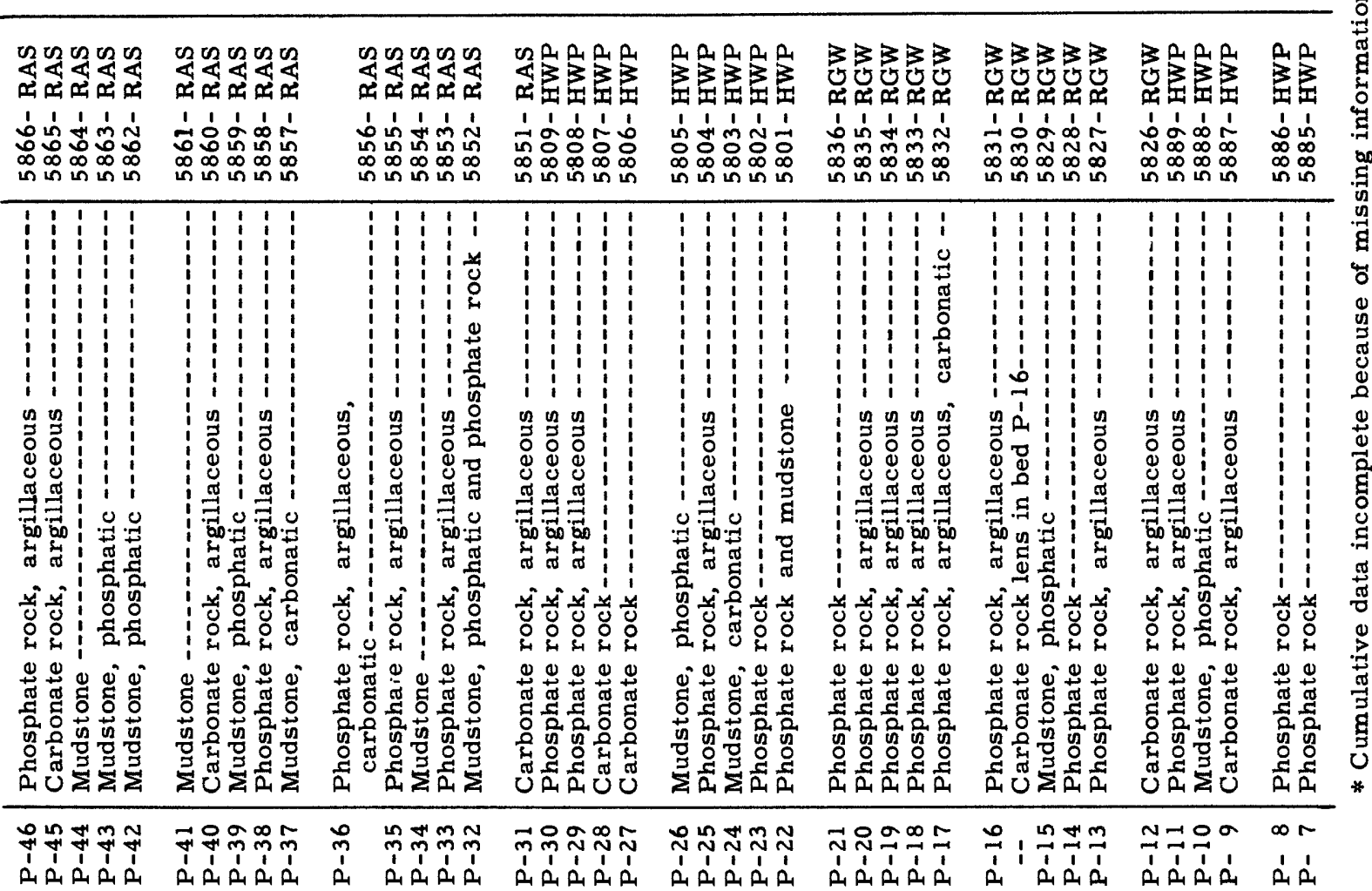




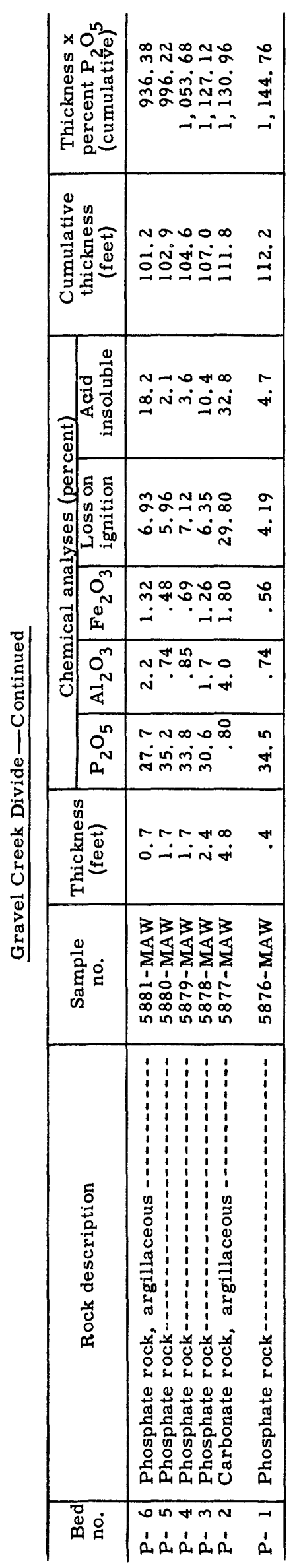




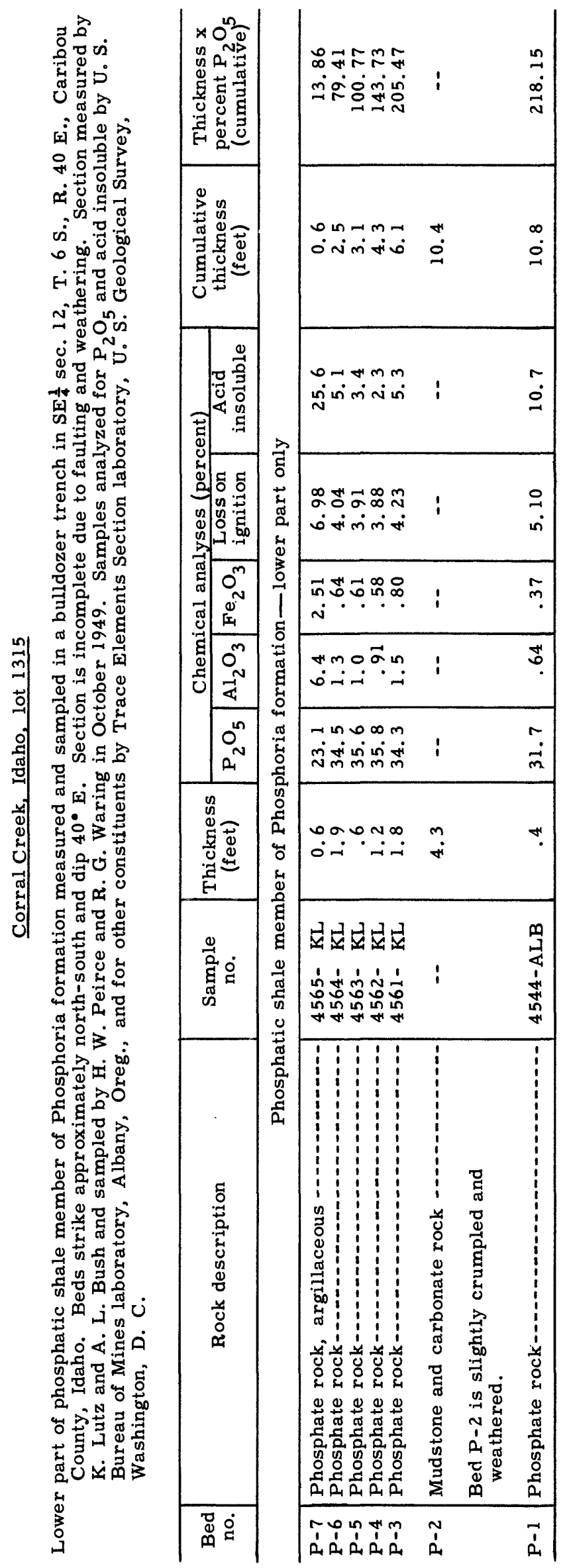




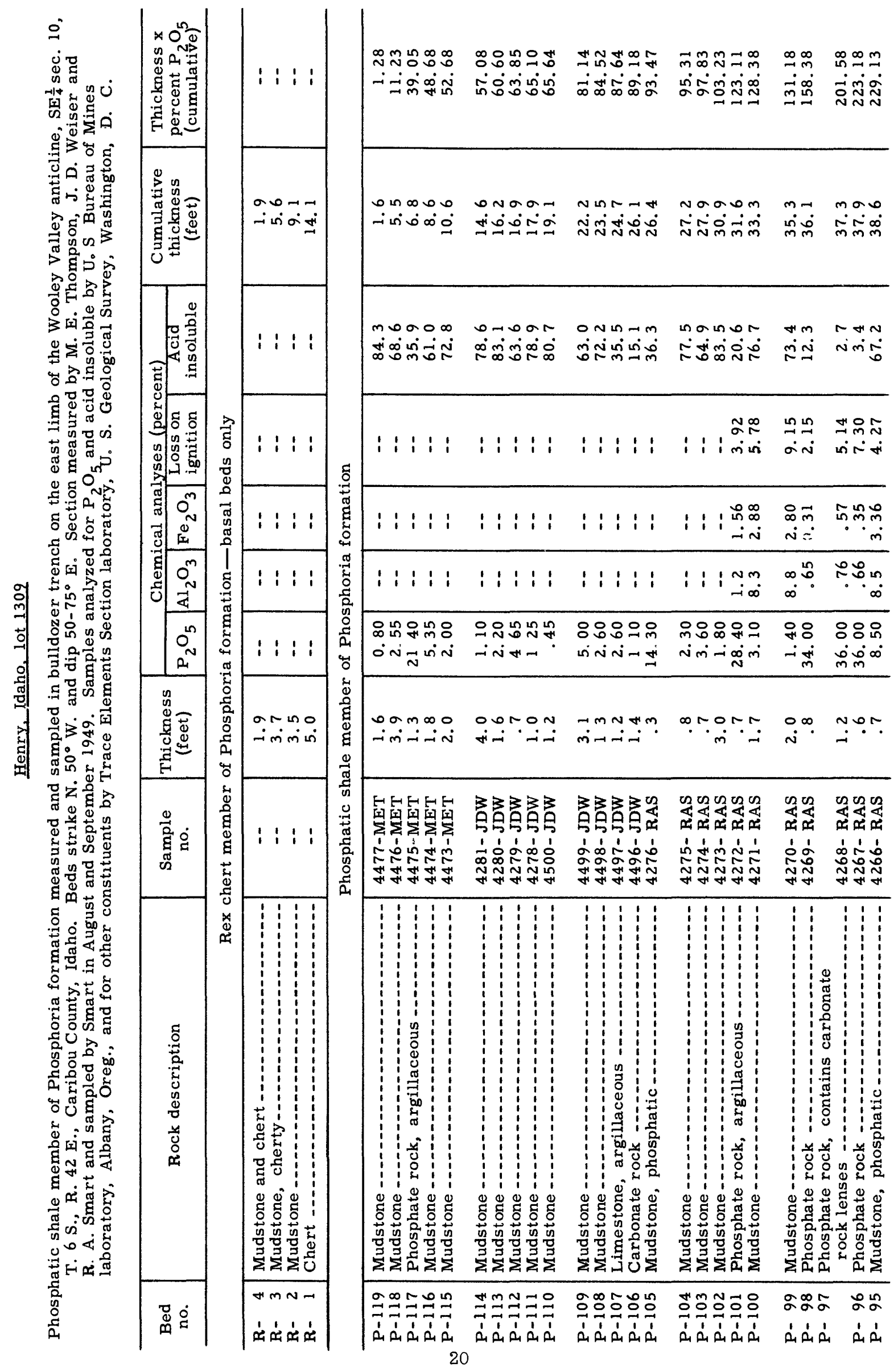




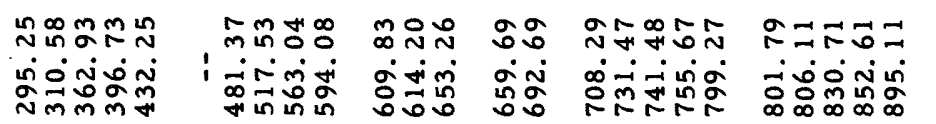

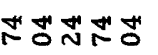

유ำ

ลิกิ์

농요

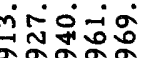

ลㅇํำ

i่

ini-

000이 $=$

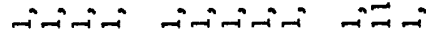

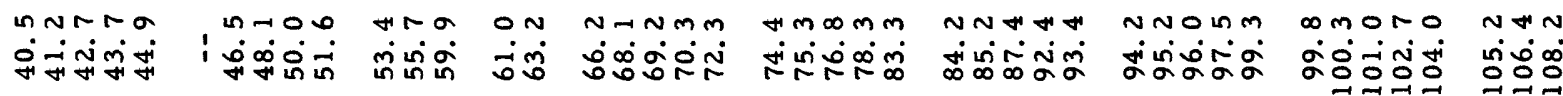

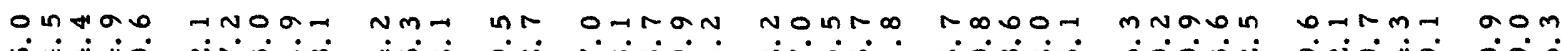

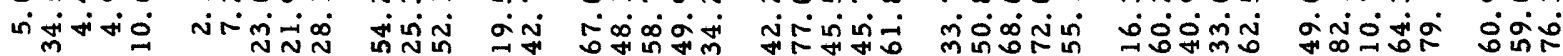

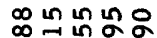

ก่

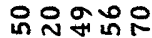

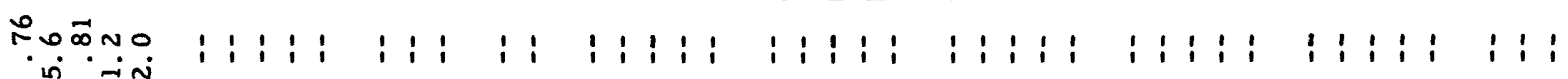

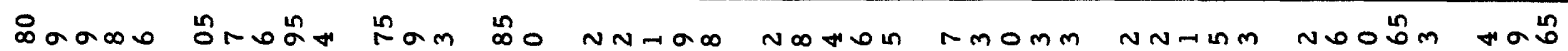

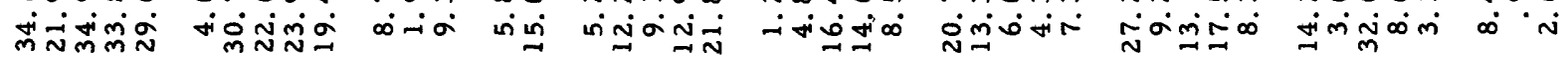

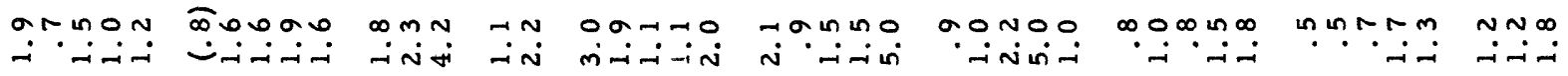

\begin{tabular}{|c|c|c|c|c|c|c|c|c|c|}
\hline 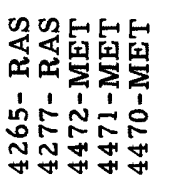 & 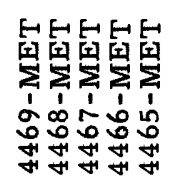 & 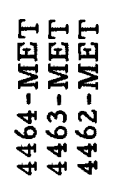 & 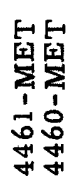 & 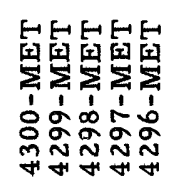 & 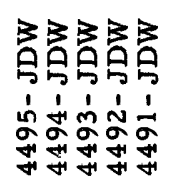 & 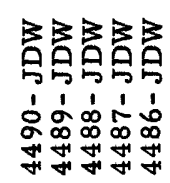 & 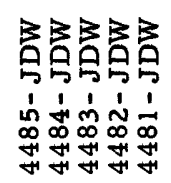 & 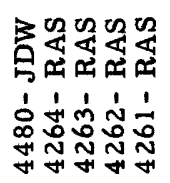 & 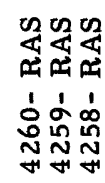 \\
\hline 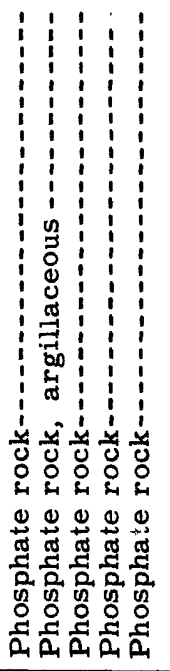 & 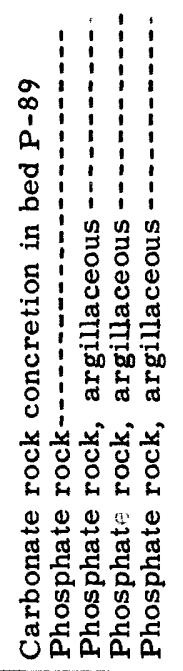 & 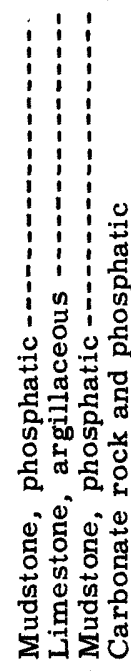 & 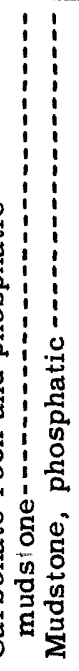 & 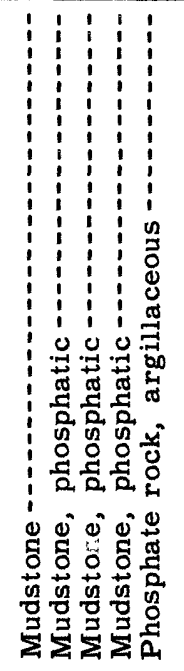 & 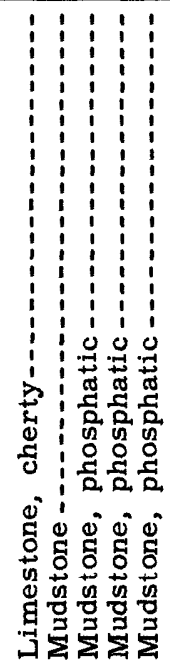 & 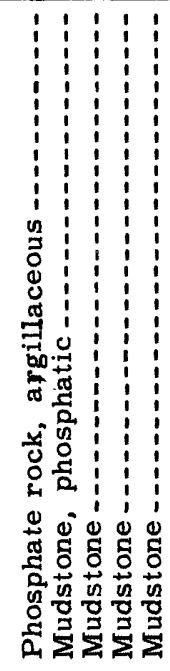 & 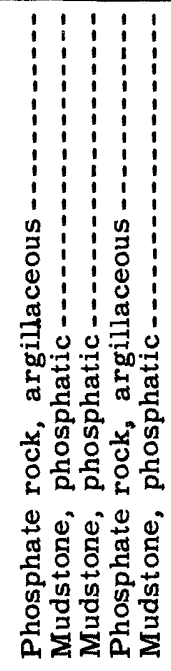 & 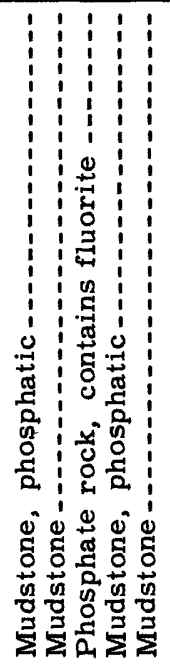 & 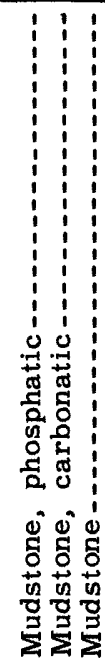 \\
\hline$\dot{n}_{1}$ & 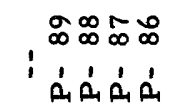 & $a_{1} 0$ & $\begin{array}{l}\vec{\infty} \\
\dot{\alpha}\end{array}$ & 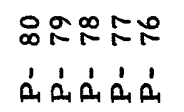 & $\dot{n}_{1} \dot{n}_{0}$ & $\begin{array}{l}5: 0 \\
\dot{a}_{1}^{\prime} \\
\dot{n}_{1}\end{array}$ & $\begin{array}{l}\text { مैं } \\
\dot{A}_{1} \dot{1}\end{array}$ & 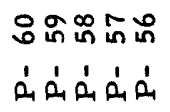 & $D_{1}$ \\
\hline
\end{tabular}




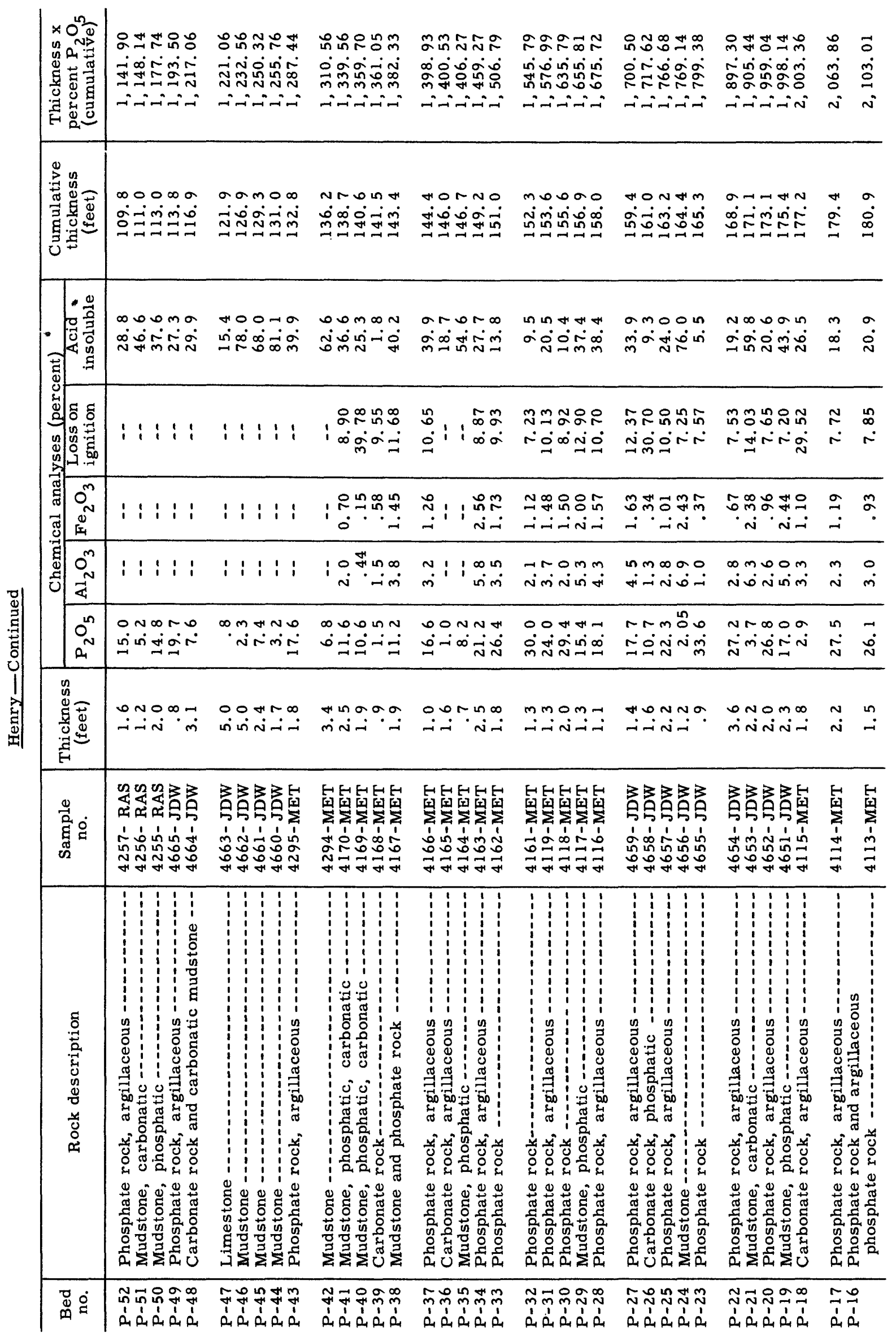




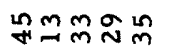

요을

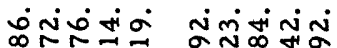

㲾的

Nininin nininin

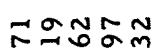
मiंmi்

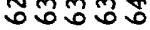
Nivinivi

$\operatorname{nin} N$ in 0

$\operatorname{rnON}$

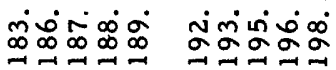

Novor

aㅇํㅇ n

NNN

\begin{tabular}{|c|c|c|}
\hline 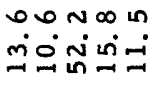 & $\begin{array}{l}0 \text { in } m \text { in } \\
\dot{m} \dot{m} \dot{m} m\end{array}$ & 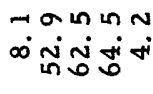 \\
\hline
\end{tabular}

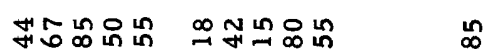

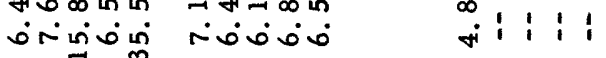

\begin{tabular}{|c|c|c|}
\hline 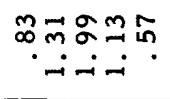 & 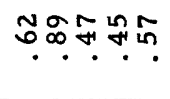 & 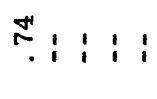 \\
\hline 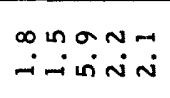 & 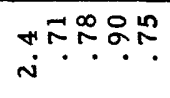 & 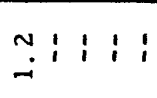 \\
\hline 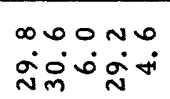 & 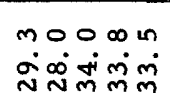 & 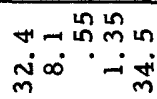 \\
\hline
\end{tabular}

\begin{tabular}{|c|c|c|c|}
\hline 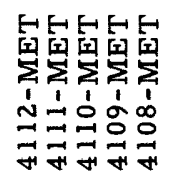 & 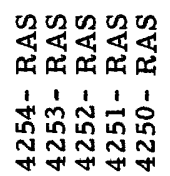 & & 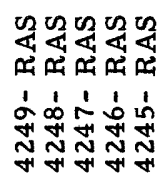 \\
\hline 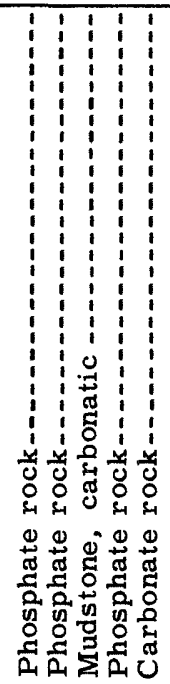 & $\begin{array}{l}1 \\
1 \\
1\end{array}$ & 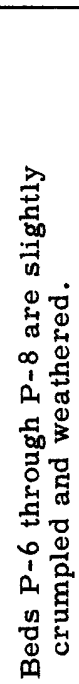 & 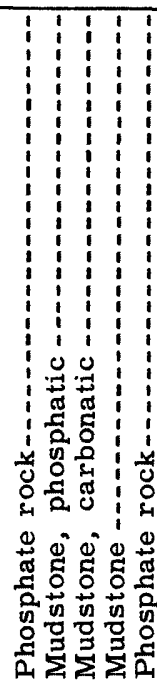 \\
\hline 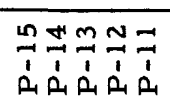 & 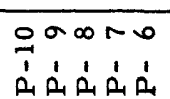 & & $\begin{array}{l}n+m N=1 \\
n^{\prime} n^{\prime} p^{\prime} p^{\prime}\end{array}$ \\
\hline
\end{tabular}




\begin{tabular}{|c|c|c|c|c|c|c|c|c|c|c|}
\hline 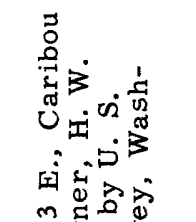 & 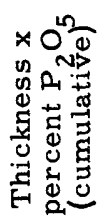 & & 1 & & 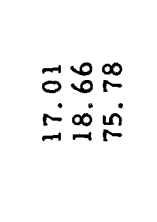 & 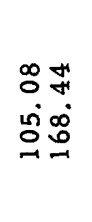 & 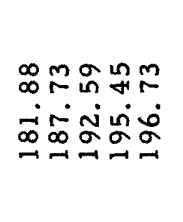 & 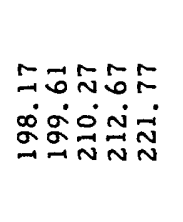 & 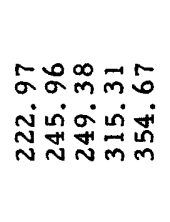 & 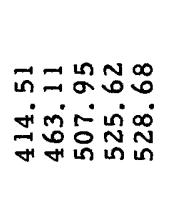 \\
\hline 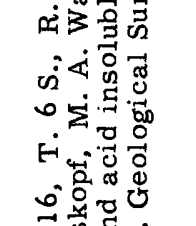 & 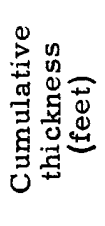 & & $\left|\begin{array}{r}\because n \\
0-i\end{array}\right|$ & & $\begin{array}{l}\sim N \infty \\
\because N M\end{array}$ & $\begin{array}{l}\infty \\
+\infty \\
\dot{\gamma}=0\end{array}$ & 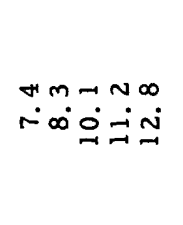 & 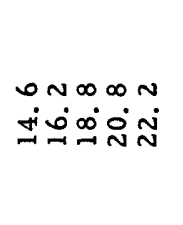 & 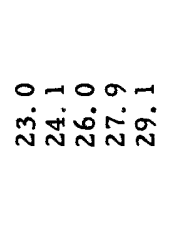 & $\begin{array}{l}m-10 \sigma 0 \\
\dot{m} m \dot{m i n}\end{array}$ \\
\hline 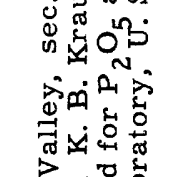 & 佘 & & 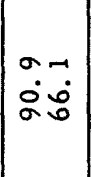 & & 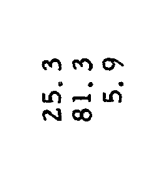 & $\begin{array}{l}\infty \\
\dot{0} \\
\dot{0}\end{array}$ & 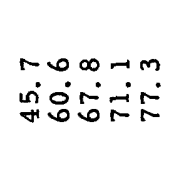 & 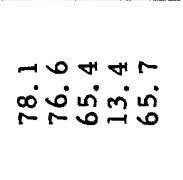 & 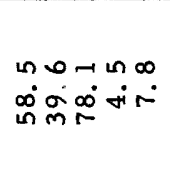 & 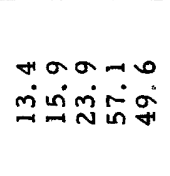 \\
\hline 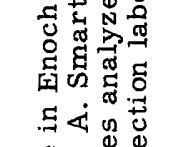 & $\left.\begin{array}{lll} & \\
0 \\
0 \\
0\end{array}\right)$ & $\begin{array}{l}\vec{\Xi} \\
\text { : } \\
\text { n }\end{array}$ & i i & $\tilde{\delta}$ & : & $\begin{array}{l}\text { 윰 } \\
\text { rim }\end{array}$ & i $1: 119$ & 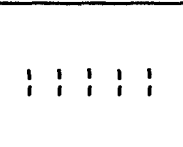 & i : : im min & $\begin{array}{l}\sigma_{\infty}^{\infty} \\
0_{0}^{\circ}\end{array}$ \\
\hline 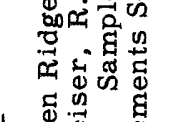 & 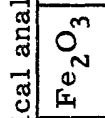 & 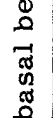 & i & 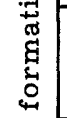 & $1: \begin{array}{l}\stackrel{0}{1} \\
0\end{array}$ & 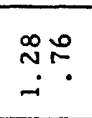 & $\begin{array}{ll:l}1 & 1 & 1\end{array}$ & 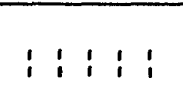 & $i: i^{+} \stackrel{\infty}{\sharp}$ & 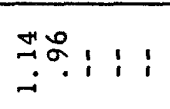 \\
\hline 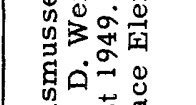 & 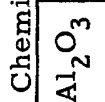 & $\underset{\Xi}{T}$ & ! & $\begin{array}{l}\frac{\pi}{\pi} \\
0 \\
\frac{a}{2}\end{array}$ & $1: \frac{0}{0}$ & 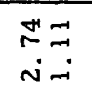 & $1: 1: 1:$ & i $i 1: i$ & i : i & $\begin{array}{l}\dot{B}_{\infty}^{m}: \\
\dot{m} \mathbf{N}\end{array}$ \\
\hline 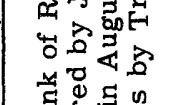 & $0_{n}^{\text {in }}$ & 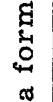 & $\mid \begin{array}{c}N \\
\vdots \\
O\end{array}$ & $\begin{array}{l}0 \\
\frac{g}{R} \\
4 \\
0\end{array}$ & 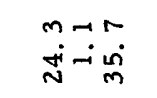 & $\begin{array}{l}m N \\
\dot{N} \\
\dot{N} m\end{array}$ & 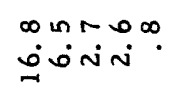 & 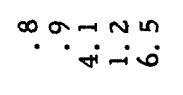 & 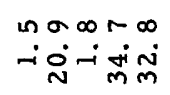 & 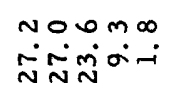 \\
\hline 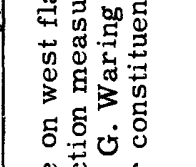 & 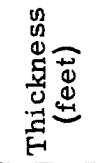 & 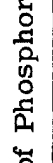 & $\because \infty$ & 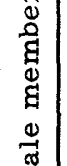 & $\because \ddot{0}$ & $\stackrel{0}{-\infty}$ & $\stackrel{\infty}{\cdot} \cdot \stackrel{\infty}{\cdot}$ & 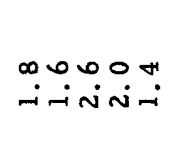 & 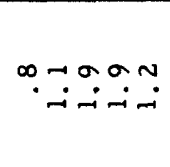 & 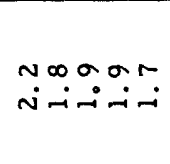 \\
\hline 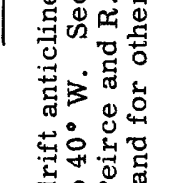 & 善。 & 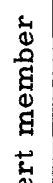 & 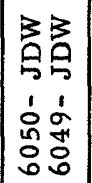 & 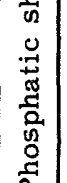 & 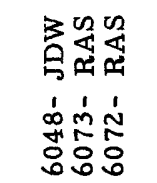 & 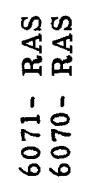 & 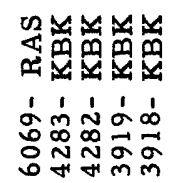 & 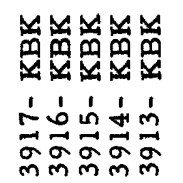 & 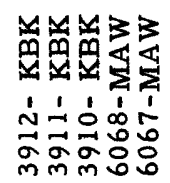 & 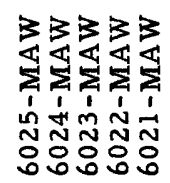 \\
\hline 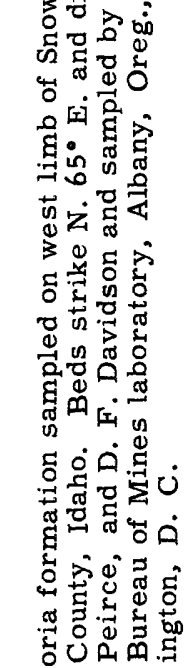 & 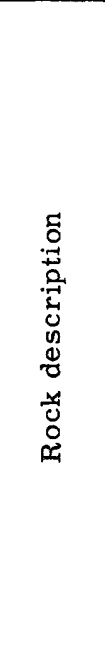 & $\stackrel{x}{\infty}$ & 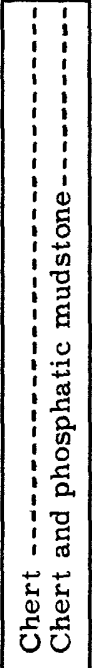 & & 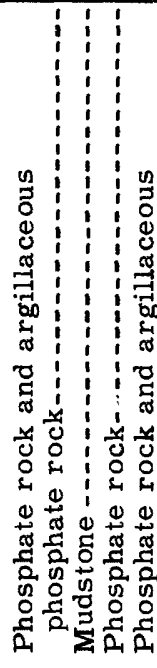 & $\begin{array}{ll}0 \\
0 \\
0 \\
0 \\
0\end{array}$ & 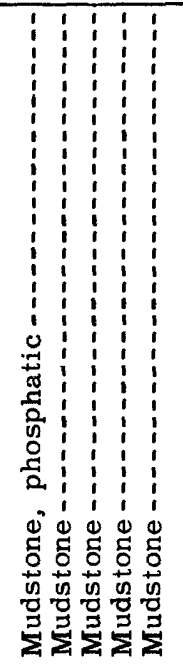 & 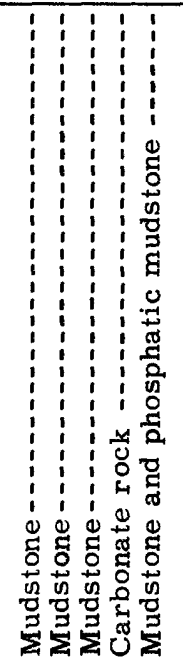 & 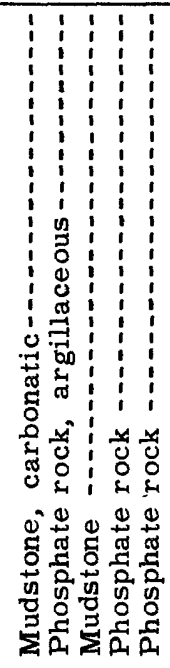 & 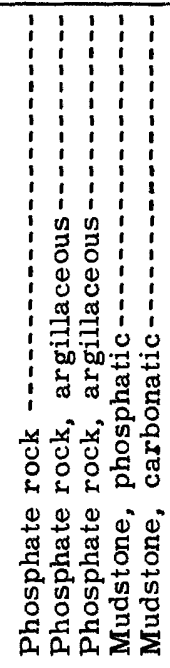 \\
\hline $\begin{array}{l}\text { के } \\
0 \\
0 \\
\frac{1}{2}\end{array}$ & Dें & & 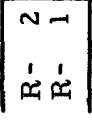 & & 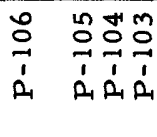 & $\begin{array}{c}0 \\
0 \\
0 \\
0 \\
24 \\
24\end{array}$ & 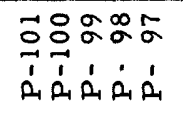 & 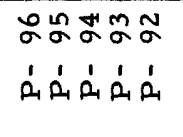 & 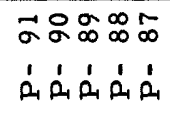 & 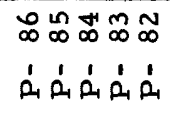 \\
\hline
\end{tabular}




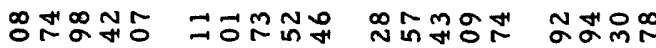

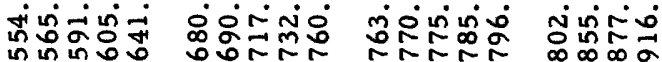

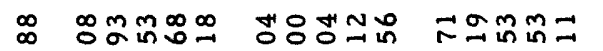

จำำนกำก

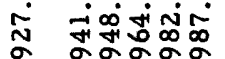

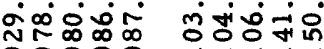

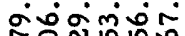

00000 에그NNNNNN

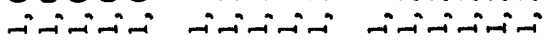

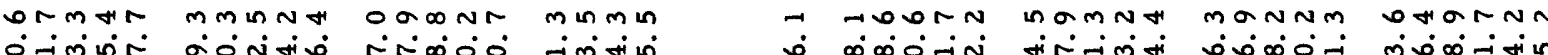

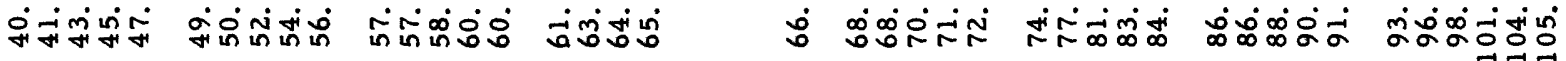

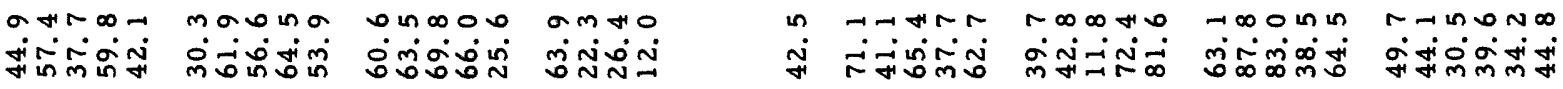

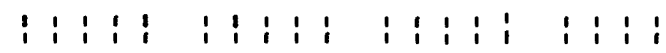

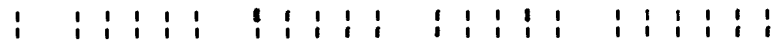

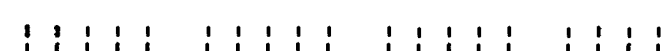

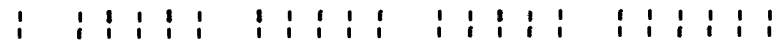

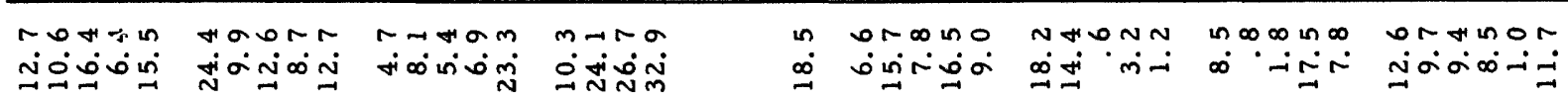

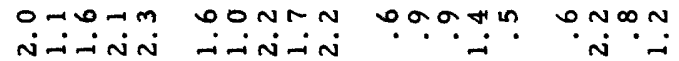

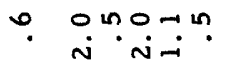

mathon anom

$m \infty$ in $\infty$ in 0

\begin{tabular}{|c|c|c|c|c|c|c|c|c|}
\hline 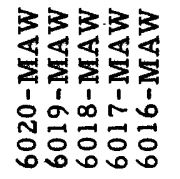 & 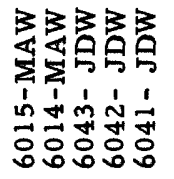 & 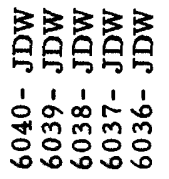 & 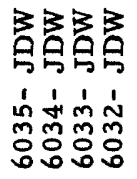 & $\begin{array}{l}3 \\
0 \\
1 \\
0 \\
0 \\
0\end{array}$ & 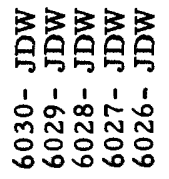 & 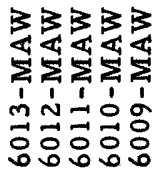 & 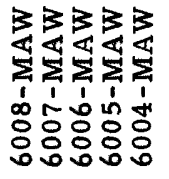 & 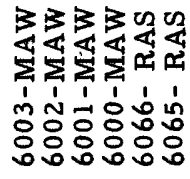 \\
\hline
\end{tabular}

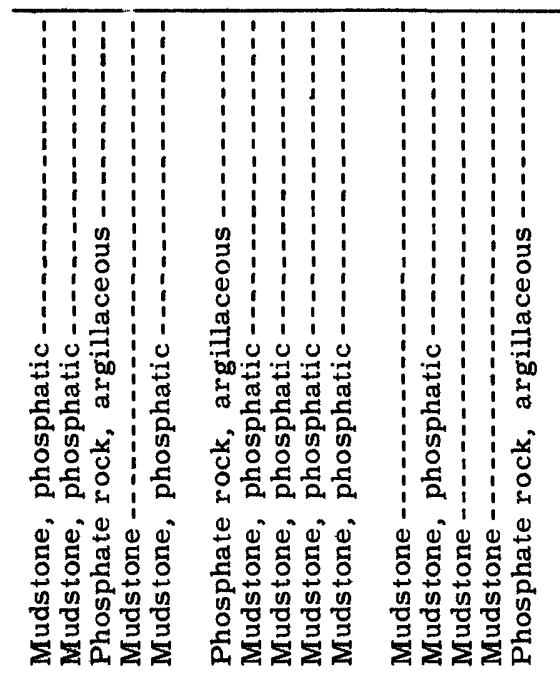

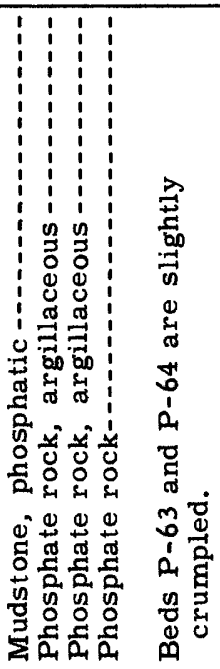

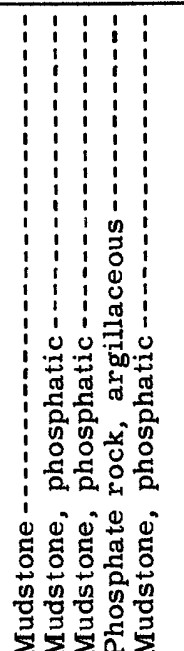

क

a

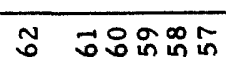

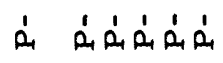

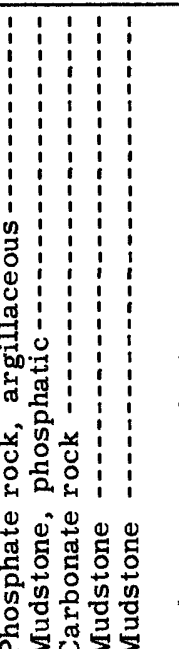

क里它空

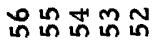

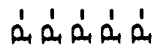

1.

元

, 응

| 刃ु

㱏

䒕 สี

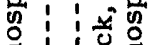

责: :

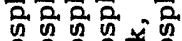

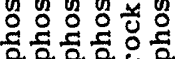

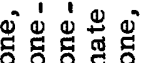

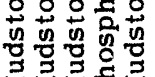

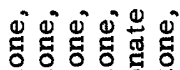

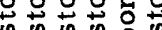

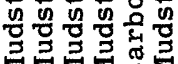

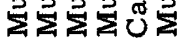

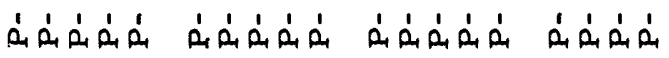




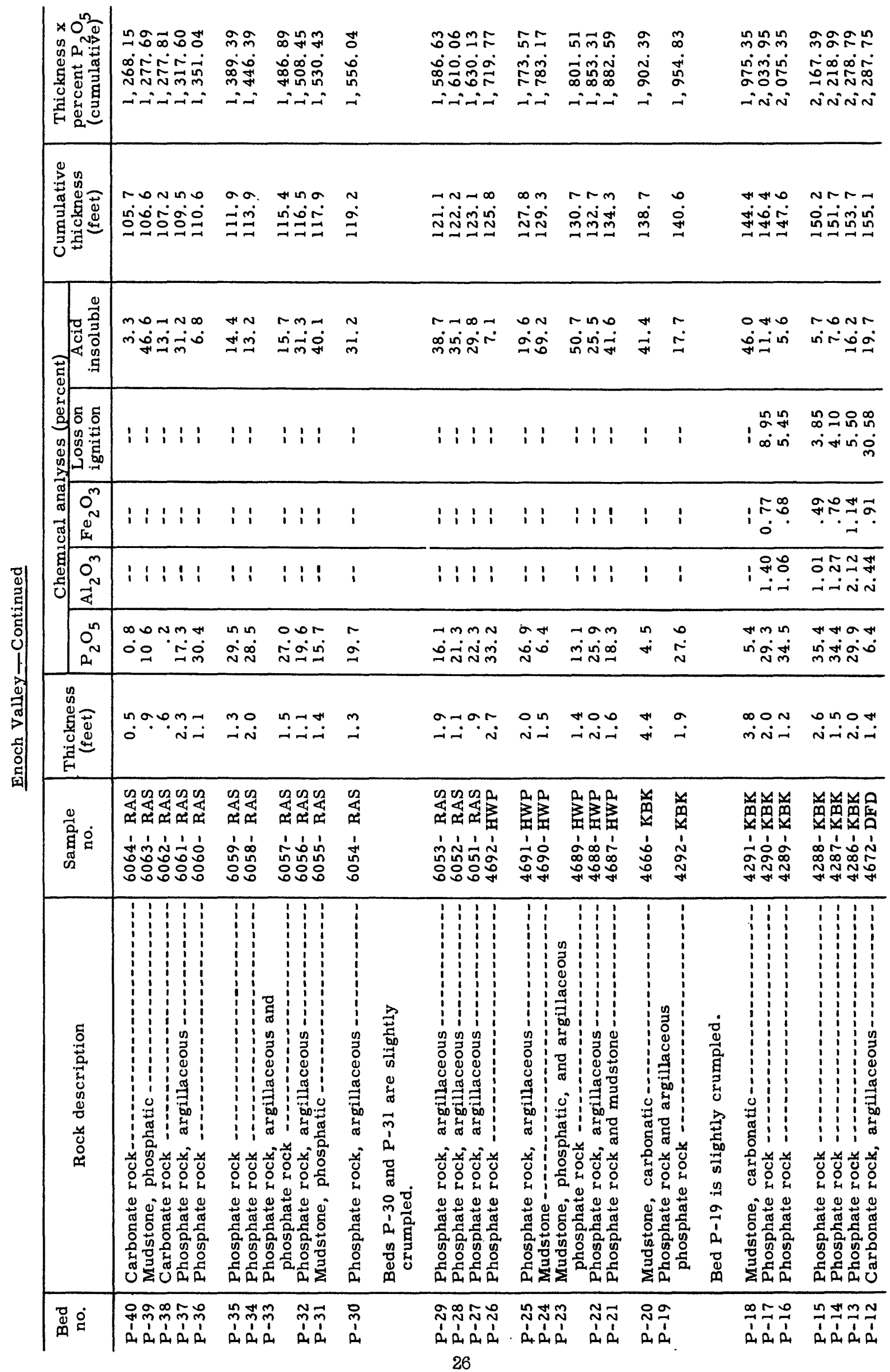




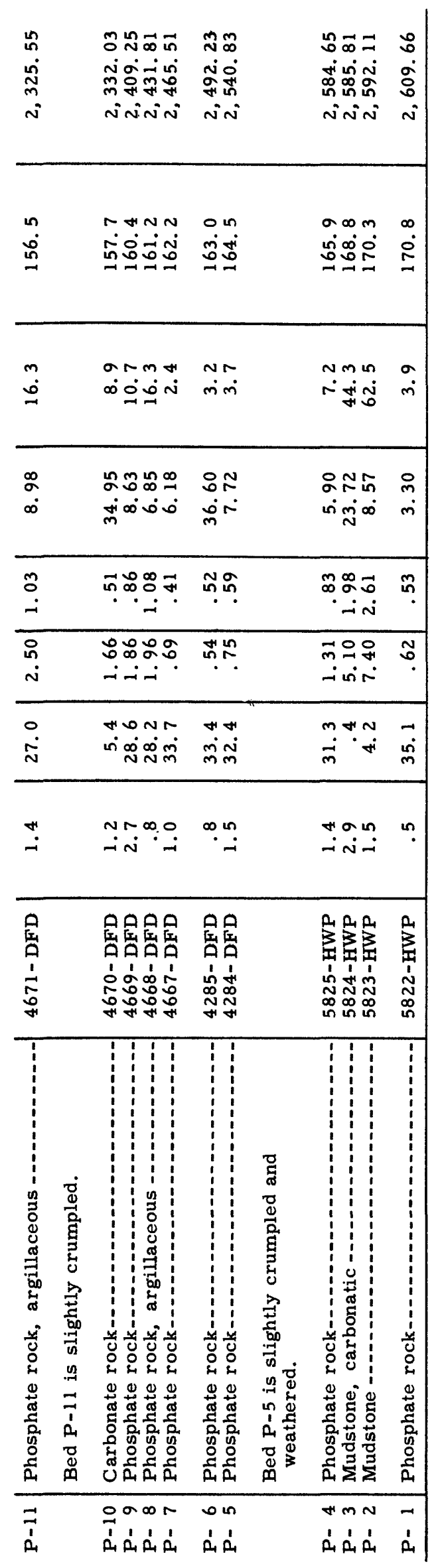




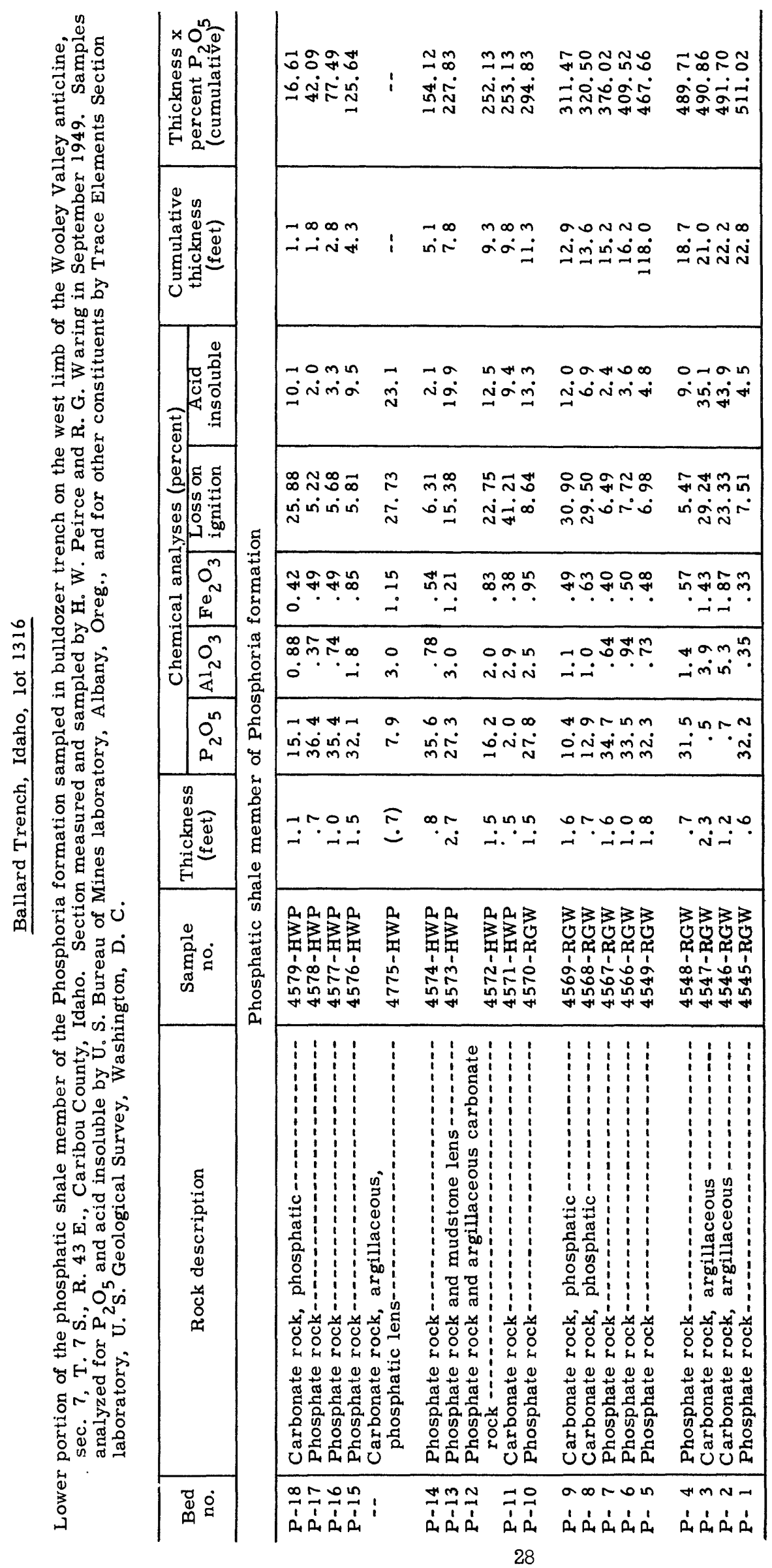

